\title{
A Quasi-Steady State Model of a Singularly Perturbed Wind Turbine with an Induction Generator and Nonlinear Frictions: A Bond Graph Approach
}

\author{
Aaron Padilla-Garcia (D, ${ }^{1}$ Gilberto Gonzalez-Avalos ${ }^{D},{ }^{1}$ Noe Barrera-Gallegos, ${ }^{2}$ \\ and Gerardo Ayala-Jaimes ${ }^{3}$ \\ ${ }^{1}$ Faculty of Electrical Engineering, University of Michoacan, Morelia, Mexico \\ ${ }^{2}$ Faculty of Mechanical Engineering, University of Michoacan, Morelia, Mexico \\ ${ }^{3}$ Faculty of Sciences of Engineering and Technology, Autonomous University of Baja California, Tijuana, Mexico
}

Correspondence should be addressed to Aaron Padilla-Garcia; joseaaronp@gmail.com

Received 20 December 2019; Accepted 11 February 2020; Published 1 April 2020

Academic Editor: Abdullah A. Kendoush

Copyright (c) 2020 Aaron Padilla-Garcia et al. This is an open access article distributed under the Creative Commons Attribution License, which permits unrestricted use, distribution, and reproduction in any medium, provided the original work is properly cited.

\begin{abstract}
The modelling in bond graph of a class of nonlinear systems with singular perturbations is presented. The class of nonlinear systems modelled by bond graphs is defined by the product of state variables and nonlinear dissipation elements. In order to obtain the mathematical model of the singularly perturbed nonlinear systems, a lemma based on the junction structure of the bond graph with a preferred integral causality assignment is proposed. The quasi-steady state model of the system is obtained by assigning a derivative causality to the storage elements for the fast dynamics and an integral causality to the storage elements for the slow dynamics. The proposed methodology to a wind turbine connected to an induction generator is applied. Simulation results of the exact and reduced models of this case study are shown.
\end{abstract}

\section{Introduction}

A common simplification used in developing control and system analysis is the reduction of system order. Hence, the application of the singular perturbation methods for separation of different time scales can be applied [1,2]. Other forms of model reduction in the literature use the concept of activity which is a measurement of energy in the elements of the system and excluding those with lower energetic activity [3]. Systems with singular perturbation are characterized by a small parameter multiplying the derivatives of the state variables called fast dynamics compared to the other variables called slow variables of the system. When the small parameter becomes zero the order of the system can be reduced $[1,2]$. In the last years, the amount of electrical energy produced by wind energy has been increased. Hence, the wind turbines have great influence in the dynamics of electrical power systems. There is a considerable amount of papers about modelling, analysis, and control of wind turbines, and it is important to make a difference between the contribution of this paper and the state of the art of wind turbines models.

In [4], the modelling and simulation of the wind turbine using two dynamic controllers are designed. In [5], the dynamic behavior of a typical wind turbine connected to the grid is described by using Matlab/Simulink with a modular structure. The use of energy and reliability of wind turbines depending on the applied control strategy are proved in [6].

One important part of the wind turbine is the electrical machinery. The induction machine has been commonly used in wind turbines and its model is important to describe the dynamic of the energy produced. Most books and papers present the traditional mathematical models of the induction machine. Procedures for estimation and control of induction machines are provided in [7]. A relatively easy way to present the model of induction machines in electrical 
power systems and industrial electronics is shown in [8]. Different control strategies for the induction machine are given in [9].

The need to have computational tools for the simulation of induction machines has been presented for many years. The computational methods for a representation of a symmetrical machine in the arbitrary reference frame are described in [10]. The electrical machines' control and the determination of the steady state response using the transformation to the reference frame are described in [9]. It is well known that the wind turbines models have different energy domains. Hence, these models can be analyzed through the bond graph methodology which provides a direct and graphic alternative. The main characteristics of the bond graph theory are [11-15]

(1) Bond graph theory provides a formal and systematic language for modelling dynamic systems

(2) A bond graph permits to represent systems with different energy domains

(3) Structural properties (stability, controllability, and observability) of a system can be obtained

Some papers determining bond graphs models of induction machines have been published. A bond graph of an induction machine considering mechanical components has been developed in [16]. The wind turbine models determine a set of high-order nonlinear differential equations. Also, an electrical power system with a wind turbine has electrical and mechanical components. Hence, the singular perturbation theory to this system can be applied. A singularly perturbed system is characterized by having slow and fast dynamics. Normally, the fast dynamics represent the electrical state variables and the mechanical state variables are the slow dynamics of an electromechanical system. Then, reduced systems applying the singularly perturbed theory can be obtained. Some papers using singular perturbations methods for bond graph models have been published. A useful bond graph methodology to simplify the analysis of linear systems of two time scales is proposed in [17]. In [18], it is introduced as a methodology to separate the slow and fast dynamics of a system and these dynamics can be estimated by causal loop gains. The direct determination of the quasi-steady state model of a system with singular perturbations in the physical domain is presented in [19]. A bond graph model for a closed-loop system with singular perturbations is proposed in [20]. A procedure to obtain approximate bond graph models for LTI MIMO systems with singular perturbations based on new junction structures is presented in [21].

The quasi-steady state model for a class of nonlinear systems with singular perturbations modelled by bond graphs is proposed in [22]. Hence, this paper is based on the previous work developed in [22]. The contribution of this paper is to analyze another class of nonlinear systems with singular perturbations with respect to [22] in a bond graph approach. The class of nonlinear systems of this paper consists of the product of state variables with nonlinear dissipation elements. Then, the quasi-steady state model is obtained from a bond graph whose storage elements of the slow dynamics have an integral causality assignment and the storage elements that represent the fast dynamics, and a derivative causality is assigned.

In this paper, an interesting case study described by the connection of a wind turbine to an induction generator is proposed. The complete system is modelled by bond graphs and the quasi-steady state model of the nonlinear system with singular perturbations applying the proposed methodology is determined. This paper has, in common, the use of the causality assignment to obtain reduced models, with respect to [20-22]. However, the papers [20, 21] are dedicated to open and closed-loop linear systems, respectively. Also, in this paper, the class of nonlinear systems has been extended with respect to [22]. The junction structure was originally presented by [14]. It was also used for different purposes in [15, 23-25]. In this paper, the junction structure is used to extend the singular perturbation methodology to a class of nonlinear systems. The organization of the paper is as follows. The singular perturbation theory in the traditional approach is described in Section 2. The class of nonlinear systems with singular perturbations in a bond graph approach is proposed in Section 3. The quasi-steady state model of the nonlinear system in the physical domain is presented in Section 4. The proposed methodology is applied to the connection of a wind turbine and an induction machine in Section 5. The conclusions are given in Section 6.

\section{The Standard Singular Perturbation Model}

Consider the singularly perturbed system model:

$$
\begin{aligned}
\dot{x}_{1} & =f\left(x_{1}, x_{2}, u, t\right), \quad x_{1}\left(t_{0}\right)=x_{1}^{0}, \\
\varepsilon \dot{x}_{2} & =g\left(x_{1}, x_{2}, u, t\right), \quad x_{2}\left(\mathrm{t}_{0}\right)=x_{2}^{0},
\end{aligned}
$$

where $x_{1}$ and $x_{2}$ are $n$ - and $m$-dimensional state vectors, respectively, $u$ is an $r$-dimensional control vector, $t$ is the time, and $\varepsilon$ is a small positive parameter; $f$ and $g$ are continuously differentiable with respect to all arguments. The system defined by (1) and (2) is called the full system. The reduced system of the full system is defined by setting $\varepsilon=0$ in (2):

$$
\begin{aligned}
\dot{\overline{x_{1}}} & =f\left(\overline{x_{1}}, \overline{x_{2}}, u, t\right), \\
0 & =g\left(\overline{x_{1}}, \overline{x_{2}}, u, t\right) .
\end{aligned}
$$

Assume that (4) can be solved uniquely, and this condition means the existence of the global solution of (4) is defined by $h$, for $x_{2}$, and denote this solution as

$$
\overline{x_{2}}=h\left(\overline{x_{1}}, u, t\right) \text {. }
$$

Then, $x_{2}$ can be eliminated from (3) and the equation gives the reduced system:

$$
\dot{x}_{1}=f\left(\overline{x_{1}}, h\left(\overline{x_{1}}, u, t\right), u, t\right) .
$$

The reduced system (6) plays a key role in the analysis and the design problem of the singularly perturbed system 
(1) To obtain the fast components we rewrite (1) and (2) in the fast scale $\tau$ :

$$
\begin{aligned}
\frac{\mathrm{d} x_{1}}{\mathrm{~d} \tau} & =\varepsilon f\left(x_{1}, x_{2}, u, t^{\prime}+\varepsilon \tau\right), \\
\frac{\mathrm{d} x_{2}}{\mathrm{~d} \tau} & =g\left(x_{1}, x_{2}, u, t^{\prime}+\varepsilon \tau\right),
\end{aligned}
$$

where

$$
\tau=\frac{t-t^{\prime}}{\varepsilon}
$$

In the limit, $\varepsilon \longrightarrow 0$. Hence, $\mathrm{d} x_{1} / \mathrm{d} \tau=0, x_{1}$ is a constant in the fast time scale, and the only fast variations are the deviations of $x_{2}$ from its quasi-steady state $\overline{x_{2}}$. Considering $x_{2 f}=x_{2}-\overline{x_{2}}$ and setting $\varepsilon=0$ in (7) and (8), we determine

$$
\frac{\mathrm{d} x_{2 f}}{\mathrm{~d} \tau}=g\left(x_{1}^{0}, x_{2}^{0}+x_{2 f}(\tau), u, t_{0}\right), \quad x_{2 f}(0)=x_{2}-\overline{x_{2}} \text {. }
$$

The fixed instant $t^{\prime}$ has been chosen to be $t_{0}$ and hence the model constants are $t_{0}, x_{1}^{0}, \overline{x_{2}^{0}}=\overline{x_{2}}\left(t_{0}\right)$, which is suitable for the fast phenomena occurring near $t_{0}$.

From (3) and (4) as the slow model and (10) as the fast model, one expects to approximate $x_{1}$ and $x_{2}$ by

$$
\begin{aligned}
& x_{1}(t) \simeq \overline{x_{1}}(t), \\
& x_{2}(t) \simeq \overline{x_{2}}(t)+x_{2 f}\left(\frac{t-t_{0}}{\varepsilon}\right),
\end{aligned}
$$

where $x_{2 f}$ is expressed in the $t$ time scale.

Finally, the eigenvalues of $\partial g / \partial x_{2}$ evaluated for $\varepsilon=0$ along $\overline{x_{1}}(t)$ have real parts smaller than a fixed negative number defined by

$$
\operatorname{Re}\left\{\frac{\partial g}{\partial x_{2}}\right\} \leq-c \leq 0 .
$$

In Section 3, the nonlinear systems with two time scales and nonlinear dissipation elements modelled by bond graphs are proposed.

\section{Bond Graph Models of Nonlinear Systems with Singular Perturbations}

Bond graph models are based on the exchange of power in a system, which is the product of an effort variable $e(t)$ and a flow variable $f(t)$ whose graphical representation in BG is a power bond shown in Figure 1 [11].

In $\mathrm{BG}$, the different physical elements of a dynamic system can be built using the following elements:

(i) 1-port active elements or sources denoted by $\left(\mathrm{MS}_{e}, \mathrm{MS}_{f}\right)$

(ii) 1-port passive elements denoted by $(R, C, I)$ which are resistances, capacitances, and inertias

(iii) 2-port elements denoted by (MTF, MGY) representing modulated transformers and gyrators

$$
\stackrel{e(t)}{f(t)}
$$

Figure 1: A bond.

(iv) Junction elements denoted by $(0,1)$ that determine the different connections of the elements

A diagram of a Bond Graph in an integral causality assignment $[11,12,14]$ (NBGI) containing different elements of a nonlinear system is shown in Figure 2. The class of nonlinear systems of this paper is obtained by using MTF and MGY modulated by state variables which represents the product of state variables and nonlinear constitutive relations for the dissipation elements.

The key vectors according to Figure 2 are the following:

(i) $x_{1}(t) \in \mathfrak{R}^{n}$ and $x_{2}(t) \in \mathfrak{R}^{m}$ are the energy variables $p(t)$ and $q(t)$ related to $I$ and $C$ storage elements in integral causality for the slow and fast dynamics, respectively

(ii) $z_{1}(t) \in \mathfrak{R}^{n}$ and $z_{2}(t) \in \mathfrak{R}^{m}$ are the coenergy vectors of the storage elements for the slow and fast dynamics, respectively

(iii) $D_{\text {in }}^{l}(t) \in \mathfrak{R}^{r 1}$ and $D_{\text {out }}^{l}(t) \in \mathfrak{R}^{r 1}$ are the mixture of $e(t)$ and $f(t)$ indicating the power exchanges of the linear dissipation field and the junction structure

(iv) $D_{\text {in }}^{n l}(t) \in \mathfrak{R}^{r 2}$ and $D_{\text {out }}^{n l}(t) \in \Re^{r 2}$ represent the power exchanges of the nonlinear dissipation field and the junction structure

(v) $u(t) \in \mathfrak{R}^{p}$ denotes the plant input

The constitutive relationship of the storage field for the slow dynamics is

$$
z_{1}(t)=F_{1} x_{1}(t)
$$

for the fast dynamics is

$$
z_{2}(t)=F_{2} x_{2}(t)
$$

and for the linear dissipation field is

$$
D_{\text {out }}^{l}(t)=L D_{\text {in }}^{l}(t) \text {. }
$$

The constitutive relationship of the nonlinear dissipation field can be obtained when a resistor has variation with respect to any slow dynamics, for example, the friction where the speeds of rotation of the mechanical parts for a wind turbine by this phenomenon are affected. Hence, this dissipation element changes its constitutive relationship depending of the velocity which is a slow dynamic. Then, the constitutive relationship of the nonlinear dissipation field can be defined by

$$
D_{\text {out }}^{n l}(t)=\phi_{L}\left(x_{1}\right) D_{\text {in }}^{n l}(t)
$$

where $\phi_{L}\left(x_{1}\right)$ contains the nonlinear functions in terms of $x_{1}$.

Lemma 1. Consider a bond graph model of a class of nonlinear systems defined by the product of the state variables and 


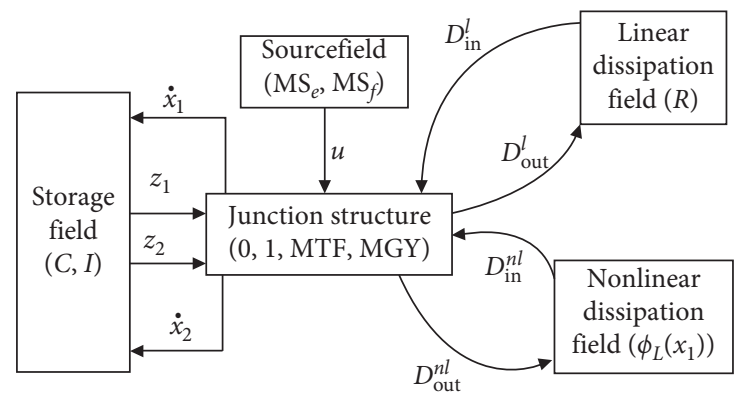

FIGURE 2: Bond graph of a class of nonlinear systems with integral causality.

dissipation elements with a nonlinear constitutive relationship of the type $D_{\text {out }}^{n l}(t)=\phi_{L}\left(x_{1}\right) D_{\text {in }}^{n l}(t)$, where the storage elements of both dynamics have an integral causality assignment (NBGI) whose junction structure is defined by by

$$
\left[\begin{array}{c}
\dot{x_{1}}(t) \\
\dot{x_{2}}(t) \\
\hline D_{\text {in }}^{l}(t) \\
D_{\text {in }}^{n l}(t)
\end{array}\right]=\left[\begin{array}{ll|ll|l}
S_{11}^{11}(x) & S_{11}^{12}(x) & S_{12}^{11}(x) & S_{12}^{12}(x) & S_{13}^{11}(x) \\
S_{11}^{21}(x) & S_{11}^{22}(x) & S_{12}^{21}(x) & S_{12}^{22}(x) & S_{13}^{21}(x) \\
\hline S_{21}^{11}(x) & S_{21}^{12}(x) & S_{22}^{11}(x) & S_{22}^{12}(x) & S_{23}^{11}(x) \\
S_{21}^{21}(x) & S_{21}^{22}(x) & S_{22}^{21}(x) & S_{22}^{22}(x) & S_{23}^{21}(x)
\end{array}\right]\left[\begin{array}{c}
z_{1}(t) \\
z_{2}(t) \\
\hline D_{\text {out }}^{l}(t) \\
D_{\text {out }}^{n l}(t) \\
\hline u(t)
\end{array}\right]
$$

Then, a state space representation $x \in\left(x_{1}, x_{2}\right)$ is defined

$$
\dot{x}_{1}(t)=A_{11}(x) x_{1}(t)+A_{12}(x) x_{2}(t)+B_{1}(x) u(t),
$$

$$
\varepsilon \dot{x}_{2}(t)=A_{21}(x) x_{1}(t)+A_{22}(x) x_{2}(t)+B_{2}(x) u(t),
$$

where

$$
\begin{aligned}
& A_{11}(x)=\left[S_{11}^{11}(x)+S_{12}^{11}(x) L T_{M}(x) S_{L 1}(x)+S_{12}^{12}(x) \phi_{L}(x) T_{\phi S}(x) S_{\phi 1}(x)\right] F_{1}, \\
& A_{12}(x)=\left[S_{11}^{12}(x)+S_{12}^{11}(x) L T_{M}(x) S_{L 2}(x)+S_{12}^{12}(x) \phi_{L}(x) T_{\phi S}(x) S_{\phi 2}(x)\right] F_{2}, \\
& A_{21}(x)=F_{2}^{-1}\left[S_{11}^{21}(x)+S_{12}^{21}(x) L T_{M}(x) S_{L 1}(x)+S_{12}^{22}(x) \phi_{L}(x) T_{\phi S}(x) S_{\phi 1}(x)\right] F_{1}, \\
& A_{22}(x)=F_{2}^{-1}\left[S_{11}^{22}(x)+S_{12}^{21}(x) L T_{M}(x) S_{L 2}(x)+S_{12}^{22}(x) \phi_{L}(x) T_{\phi S}(x) S_{\phi 2}(x)\right] F_{2}, \\
& B_{1}(x)=S_{13}^{11}(x)+S_{12}^{11}(x) L T_{M}(x) S_{B L}(x)+S_{12}^{12}(x) \phi_{L}(x) T_{\phi S}(x) S_{B \phi}(x), \\
& B_{2}(x)=F_{2}^{-1}\left[S_{13}^{21}(x)+S_{12}^{21}(x) L T_{M}(x) S_{B L}(x)+S_{12}^{22}(x) \phi_{L}(x) T_{\phi S}(x) S_{B \phi}(x)\right],
\end{aligned}
$$

with

$$
\begin{aligned}
& S_{L 1}(x)=S_{21}^{11}(x)+S_{22}^{12}(x) M_{\phi}(x) S_{21}^{21}(x), \\
& S_{L 2}(x)=S_{21}^{12}(x)+S_{22}^{12}(x) M_{\phi}(x) S_{21}^{22}(x), \\
& S_{\phi 1}(x)=S_{21}^{21}(x)+S_{22}^{21}(x) M_{L}(x) S_{21}^{11}(x), \\
& S_{\phi 2}(x)=S_{21}^{22}(x)+S_{22}^{21}(x) M_{L}(x) S_{21}^{12}(x),
\end{aligned}
$$




$$
\begin{aligned}
& S_{B L}(x)=S_{23}^{11}(x)+S_{22}^{12}(x) M_{\phi}(x) S_{23}^{21}(x), \\
& S_{B \phi}(x)=S_{23}^{21}(x)+S_{22}^{21}(x) M_{L}(x) S_{23}^{11}(x), \\
& T_{M}(x)=\left[M_{L}^{-1}(x) L-S_{22}^{12}(x) M_{\phi}(x) S_{22}^{21}(x) L\right]^{-1}, \\
& T_{\phi S}(x)=\left[M_{\phi}^{-1}(x) \phi_{L}\left(x_{1}\right)-S_{22}^{21}(x) M_{L}(x) S_{21}^{12}(x) \phi_{L}\left(x_{1}\right)\right]^{-1}, \\
& M_{L}(x)=L\left[I-S_{22}^{11}(x) L\right]^{-1}, \\
& M_{\phi}(x)=\phi_{L}\left(x_{1}\right)\left[I-S_{22}^{22}(x) \phi_{L}\left(x_{1}\right)\right]^{-1} .
\end{aligned}
$$

The proof is presented in Appendix A.

It is important to know that the submatrices of the junction structure $S_{i j}^{p q}(x)$ with $i, j, p, q=1,2,3$ and $M(x)$ can yield singularities problem due to the numerical values for the elements of the system.

Also, it is necessary to validate the assumption in (12) for the particular class of nonlinear systems given in (20) and (21); then, from (2) and (21), the nonlinear function of the fast dynamics is

$$
g=A_{21}(x) x_{1}(t)+A_{22}(x) x_{2}(t)+B_{2}(x) u(t) .
$$

Then, (14) is defined by

$$
\operatorname{Re}\left\{\frac{\partial\left[A_{21}(x) x_{1}(t)\right]}{\partial x_{2}}+\frac{\partial\left[A_{22}(x) x_{2}(t)\right]}{\partial x_{2}}+\frac{\partial\left[B_{2}(x) u(t)\right]}{\partial x_{2}}\right\} \leq 0
$$

\section{A Quasi-Steady State Model of a System with Nonlinear Dissipation Elements in the Physical Domain}

When a system is characterized as a singularly perturbed system, reduced models of this system can be obtained. It is common that the slow dynamic model of a system gives interesting properties of the dynamic performance for the complete system. Working with reduced models is simpler for analysis and synthesis compared to effort required in the analysis of large complex systems. Thus, a quasi-steady state model of a nonlinear system with singular perturbations using bond graphs is presented.
The key contribution of this paper is the causality assignment of the energy storage elements into the bond graph model. The quasi-steady state model of the system is a reduced model, considering slow and fast dynamics have reached the steady state response under their operation condition. Thus, a bond graph whose storage elements for the slow and fast dynamics have an integral and derivative causality assignment, respectively, [11,12] for a class of nonlinear systems with singular perturbations (SPNBG), determines the quasi-steady state model shown in Figure 3.

The characteristics of the bond graph of Figure 3 are

(i) The storage elements $\left(\dot{x}_{2}, z_{2}\right)$ for the fast dynamics have a derivative causality assignment

(ii) The storage elements $\left(\dot{x}_{1}, z_{1}\right)$ for the slow dynamics have an integral causality assignment

(iii) The dissipation elements $\left(D_{\text {in }}^{h l}, D_{\text {out }}^{h l}\right.$ and $\left.D_{\text {in }}^{h n l}, D_{\text {out }}^{h n l}\right)$ will have a causality assignment in order to construct a correct bond graph

The following lemma presents the main contribution of this paper.

Lemma 2. Consider a bond graph model according to the scheme of Figure 3, where the storage elements for the fast dynamics have a derivative causality assignment and the storage elements for the slow dynamics have an integral causality assignment. This bond graph represents a class of nonlinear systems with singular perturbations defined by the product of states variables and nonlinear constitutive relations of the dissipation field satisfying (16), and the junction structure is defined by

$$
\left[\begin{array}{c}
\dot{x}_{1}(t) \\
z_{2}(t) \\
\hline D_{\text {in }}^{h l}(t) \\
D_{\text {in }}^{h n l}(t)
\end{array}\right]=\left[\begin{array}{ll|ll|l}
H_{11}^{11}(x) & H_{11}^{12}(x) & H_{12}^{11}(x) & H_{12}^{12}(x) & H_{13}^{11}(x) \\
H_{11}^{21}(x) & H_{11}^{22}(x) & H_{12}^{21}(x) & H_{12}^{22}(x) & H_{13}^{21}(x) \\
\hline H_{21}^{11}(x) & H_{21}^{12}(x) & H_{22}^{11}(x) & H_{22}^{12}(x) & H_{23}^{11}(x) \\
H_{21}^{21}(x) & H_{21}^{22}(x) & H_{22}^{21}(x) & H_{22}^{22}(x) & H_{23}^{21}(x)
\end{array}\right]\left[\begin{array}{c}
z_{1}(t) \\
\dot{x_{2}}(t) \\
\hline D_{\text {out }}^{h l}(t) \\
D_{\text {out }}^{h l}(t) \\
\hline u(t)
\end{array}\right]
$$




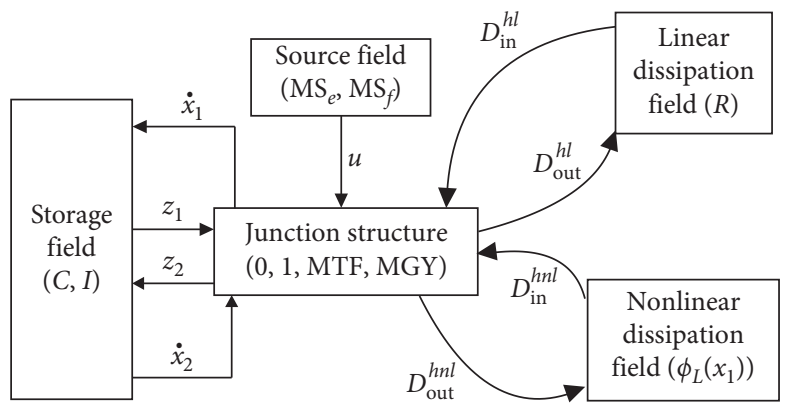

FIGURE 3: Bond graph of a class of nonlinear systems with integral and derivative causality for slow and fast dynamics, respectively.

with the constitutive relations of the dissipation field:

$$
\begin{aligned}
& D_{\text {out }}^{h l}(t)=L^{h} D_{\text {in }}^{h l}(t), \\
& D_{\text {out }}^{h n l}(t)=\phi_{L}^{h}\left(x_{1}\right) D_{\text {in }}^{h n l}(t) .
\end{aligned}
$$

Then, the quasi-steady state model for the fast dynamics is described by

$$
\overline{x_{2}}(t)=A_{21}^{h}\left(x_{1}\right) \overline{x_{1}}(t)+B_{2}^{h}\left(x_{1}\right) u(t),
$$

and for the slow dynamics is expressed by

$$
\stackrel{\dot{\bar{x}}}{x_{1}}(t)=A_{11}^{h}\left(x_{1}\right) \overline{x_{1}}(t)+B_{1}^{h}\left(x_{1}\right) u(t)
$$

where

$$
\begin{aligned}
& A_{11}^{h}\left(x_{1}\right)=\left[H_{11}^{11}\left(x_{1}\right)+H_{12}^{11}\left(x_{1}\right) L^{h} T_{Q}\left(x_{1}\right) H_{Q 1}\left(x_{1}\right)+H_{12}^{12}\left(x_{1}\right) \phi_{L}^{h}\left(x_{1}\right) T_{\phi H}\left(x_{1}\right) H_{\phi 1}\left(x_{1}\right)\right] F_{1}, \\
& A_{21}^{h}\left(x_{1}\right)=F_{2}^{-1}\left[H_{11}^{21}\left(x_{1}\right)+H_{12}^{21}\left(x_{1}\right) L^{h} T_{Q}\left(x_{1}\right) H_{Q 1}\left(x_{1}\right)+H_{12}^{22}\left(x_{1}\right) \phi_{L}^{h}\left(x_{1}\right) T_{\phi H}\left(x_{1}\right) H_{\phi 1}\left(x_{1}\right)\right] F_{1}, \\
& B_{1}^{h}\left(x_{1}\right)=H_{13}^{11}\left(x_{1}\right)+H_{12}^{11}\left(x_{1}\right) L^{h} T_{Q}\left(x_{1}\right) H_{B Q}\left(x_{1}\right)+H_{12}^{12}\left(x_{1}\right) \phi_{L}^{h}\left(x_{1}\right) T_{\phi H}\left(x_{1}\right) H_{B \phi}\left(x_{1}\right), \\
& B_{2}^{h}\left(x_{1}\right)=F_{2}^{-1}\left[H_{13}^{21}\left(x_{1}\right)+H_{12}^{21}\left(x_{1}\right) L^{h} T_{Q}\left(x_{1}\right) H_{B Q}\left(x_{1}\right)+H_{12}^{22}\left(x_{1}\right) \phi_{L}^{h}\left(x_{1}\right) T_{\phi H}\left(x_{1}\right) H_{B \phi}\left(x_{1}\right)\right],
\end{aligned}
$$

with

$$
\begin{aligned}
H_{Q 1}\left(x_{1}\right) & =H_{12}^{11}\left(x_{1}\right)+H_{22}^{12}\left(x_{1}\right) \phi_{Q}\left(x_{1}\right) H_{21}^{21}\left(x_{1}\right), \\
H_{\phi 1}\left(x_{1}\right) & =H_{21}^{21}\left(x_{1}\right)+H_{22}^{21}\left(x_{1}\right) Q\left(x_{1}\right) H_{21}^{11}\left(x_{1}\right), \\
H_{B Q}\left(x_{1}\right) & =H_{23}^{11}\left(x_{1}\right)+H_{22}^{12}\left(x_{1}\right) \phi_{Q}\left(x_{1}\right) H_{23}^{21}\left(x_{1}\right), \\
H_{B \phi}\left(x_{1}\right) & =H_{23}^{21}\left(x_{1}\right)+H_{22}^{21}\left(x_{1}\right) Q\left(x_{1}\right) H_{23}^{11}\left(x_{1}\right), \\
T_{Q}(x) & =\left[Q_{L}^{-1}(x) L^{h}-H_{22}^{12}(x) Q_{\phi}(x) H_{22}^{21}(x) L^{h}\right]^{-1}, \\
T_{\phi H}(x) & =\left[Q_{\phi}^{-1}(x) \phi_{L}\left(x_{1}\right)-H_{22}^{21}(x) Q_{L}(x) H_{21}^{12}(x) \phi_{L}^{h}\left(x_{1}\right)\right]^{-1}, \\
Q_{L}(x) & =L^{h}\left[I-H_{22}^{11}(x) L^{h}\right]^{-1}, \\
Q_{\phi}(x) & =\phi_{L}^{h}\left(x_{1}\right)\left[I-H_{22}^{22}(x) \phi_{L}^{h}\left(x_{1}\right)\right]^{-1} .
\end{aligned}
$$

The proof is presented in Appendix B.

From the second line of (38) and (41) with (14),

$x_{2}(t)=A_{21}^{h}(x) x_{1}(t)+A_{22}^{h}(x) \dot{x}_{2}(t)+B_{2}^{h}(x) u(t)$, where $\dot{x}_{2}(t)=0$ in order to get the quasi-steady state for the fast dynamics.

$$
\overline{x_{2}}(t)=A_{21}^{h}(x) \overline{x_{1}}(t)+B_{2}^{h}(x) u(t)
$$


if the terms $A_{21}^{h}(x)$ and $B_{2}^{h}(x)$ depend of both dynamics, and this algebraic system has to be solved and (41) is proved.

From (55),

$$
\begin{aligned}
\dot{x}_{2}(t)= & -\left[A_{22}^{h}(x)\right]^{-1} A_{21}^{h}(x) x_{1}(t)+\left[A_{22}^{h}(x)\right]^{-1} x_{2}(t) \\
& -\left[A_{22}^{h}(x)\right]^{-1} B_{2}^{h}(x) u(t),
\end{aligned}
$$

comparing (57) with (19), the following relations are determined:

$$
\begin{aligned}
& A_{22}^{h}(x)=A_{22}^{-1}(x) \varepsilon, \\
& A_{21}^{h}(x)=-A_{22}^{-1}(x) A_{21}(x), \\
& B_{2}^{h}(x)=-A_{22}^{-1}(x) B_{2}(x),
\end{aligned}
$$

from the first line of (38) and (42)

$$
\dot{x}_{1}(t)=A_{11}^{h}(x) x_{1}(t)+A_{12}^{h}(x) \dot{x}_{2}(t)+B_{1}^{h}(x) u(t) .
$$

By substituting (57) into (59),

$$
\begin{aligned}
\dot{x}_{1}(t)= & \left\{A_{11}^{h}(x)-A_{12}^{h}(x)\left[A_{22}^{h}(x)\right]^{-1} A_{21}^{h}(x)\right\} x_{1}(t) \\
& +A_{12}^{h}(x)\left[A_{22}^{h}(x)\right]^{-1} x_{2}(t) \\
& +\left\{B_{1}^{h}(x)-A_{12}^{h}(x)\left[A_{22}^{h}(x)\right]^{-1} B_{2}^{h}(x)\right\} u(t),
\end{aligned}
$$

comparing (18) with (60), the following relations are obtained:

$$
\begin{aligned}
& A_{12}^{h}(x)=A_{12}(x) A_{22}^{-1}(x) \varepsilon, \\
& A_{11}^{h}(x)=A_{11}(x)-A_{12}(x) A_{22}^{-1}(x) A_{21}(x), \\
& B_{1}^{h}(x)=B_{1}(x)-A_{12}(x) A_{22}^{-1}(x) B_{2}(x),
\end{aligned}
$$

from (41) with (61)-(63), the quasi-steady state model for the slow dynamics given by (42) is proved.

The matrices $Q_{L}(x)$ and $Q_{\phi}(x)$ and submatrices of the junction structure $H_{i j}^{p q}(x)$ with $i, j, p, q=1,2,3$ can determine singularities' problems due to the numerical values of the parameters of the system. This is important to detect in order to avoid stability and simulating problems. Another important condition is that $A_{22}(x)$ must be nonsingular.

The bond graph model and the quasi-steady state model of a wind turbine system are obtained in Section 5.

\section{An Induction Generator and Mechanical Models of a Wind Turbine Modelled by Bond Graphs}

In this section, the proposed methodology to a nonlinear singularly perturbed system is applied. In the electromechanical systems, the mechanical variables represent the state variables for the slow dynamics and the electrical variables are the state variables for the fast dynamics. The air friction at high speeds, as a fluid with nonlinear resistance can be considered. This mechanical phenomenon occurs when there is a relative velocity between a fluid and a body; the body experiences a drag force that opposes the relative motion and points in the direction in which the fluid flows relative to the body. The drag force is sometimes called air resistance or friction [26].

The magnitude of the drag force is related to the relative speed $v$ and the drag coefficient $C$ according to [26]:

$$
D=\frac{1}{2} C \rho A v^{2},
$$

where $\rho$ is the air density and $A$ is the effective cross-sectional area of the body. Considering all constant parameters, the following relation is obtained:

$$
c=\frac{1}{2} C \rho A \text {. }
$$

In general, the friction force is defined by

$$
F_{\text {resist }}=-b v-c v^{2}
$$

if $v \ll 1$, drop the second term; if $v \gg 1$, ignore the first one.

5.1. Schematic Diagram of the Three-Mass Equivalent Model of a Wind Turbine. A three-mass equivalent model of a wind turbine in the mechanical system is proposed in Figure 4. The first mass stands for the blades, the second mass stands for the hub, and the third mass stands for the generator including the gear box, high-speed shafts, and generator rotor [27], where $H_{b}, H_{h}$, and $H_{g}$ are the constant inertia for the blades, hub, and generator, respectively; $D_{b h}$ is the damping coefficient between the blades and hub; $D_{h g}$ is the damping coefficient between the hub and the generator rotor; $D_{b}, D_{h}$, and $D_{g}$ are the damping coefficients for the blades, hub, and generator, respectively; $K_{b h}$ and $K_{h g}$ are the stiffness of the blades and low speed shaft [28].

Considering quadratic resistance in the dissipation elements, as shown in Figure 4 , the constant $D_{g}$ is $c$ in equation (65); this constant multiplies the square of the speed of this section of a wind turbine $w_{g}^{2}=\left(p_{13} / H_{g}\right)^{2}$, where $p_{13}$ is the angular momentum.

5.2. Induction Machine Modelled by Bond Graphs. One of the most common electrical motor used in many applications is the induction motor. Figure 5 shows a scheme of a 2-pole induction machine with symmetrical 3-phase windings in the stator and rotor [29]. The stator and rotor windings are identical and sinusoidally distributed and the $s$ and $r$ subscripts denote the variables and parameters associated to the stator and rotor circuits, respectively.

The Park transformation gives an important simplification in the mathematical model of the induction machine. The property of the Park transformation is to change all 


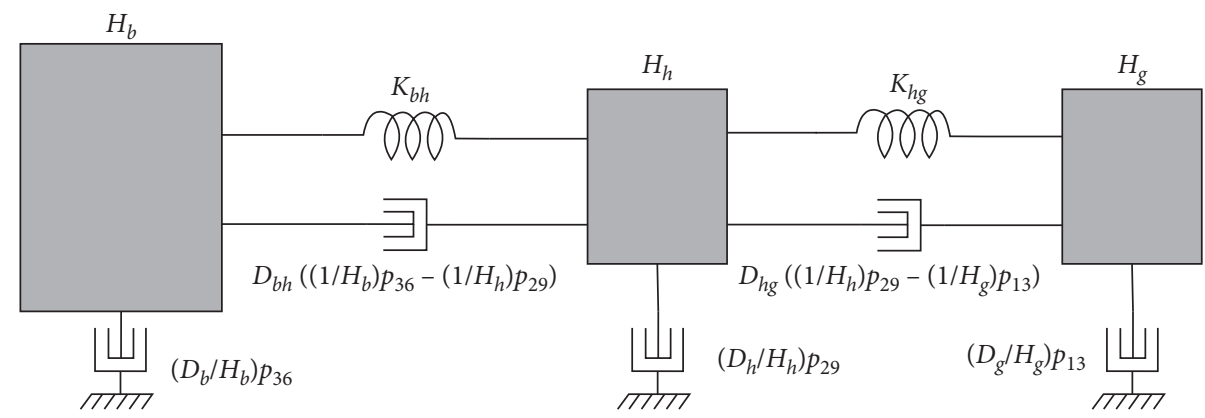

FIGURE 4: Schematic diagram of the three-mass equivalent model of a wind turbine, considering resistance quadratic in the dissipation elements.

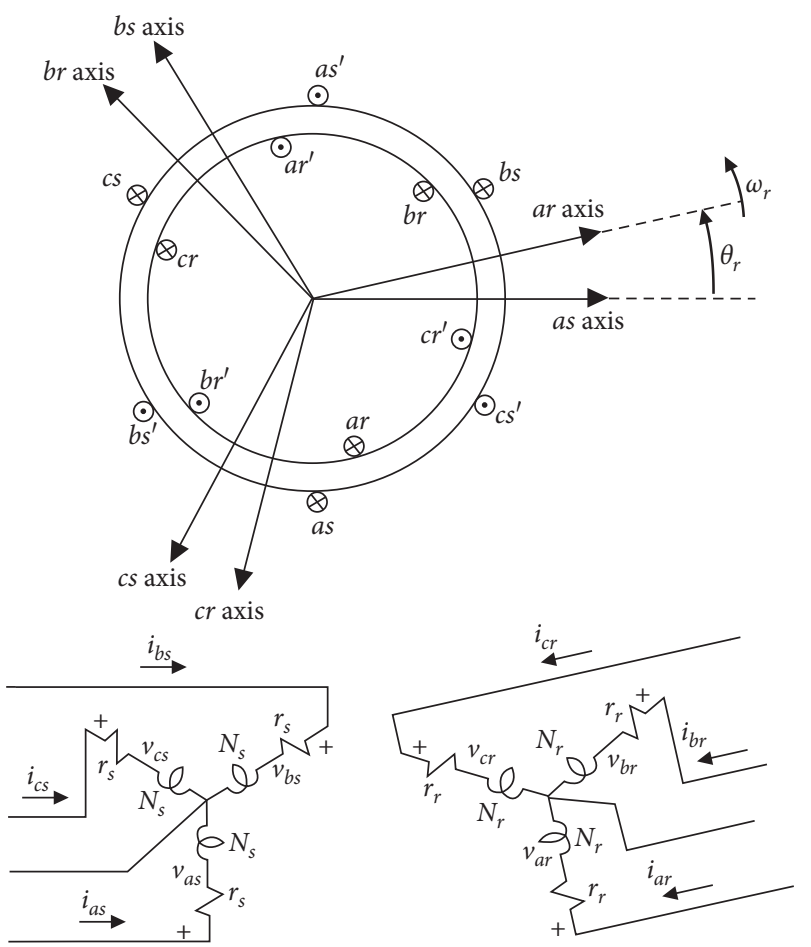

Figure 5: Schematic of symmetrical 3-phase asynchronous or induction machine.

stator variables and parameters from phases $(a, b, c)$ to new variables, the frame of reference of which moves with the rotor [29].

The Park transformation for voltages is defined by

$$
i_{d q 0}=P i_{a b c},
$$

where

$$
P=\sqrt{\frac{2}{3}}\left[\begin{array}{ccc}
\cos \theta & \cos \left(\theta-\frac{2 \pi}{3}\right) & \cos \left(\theta+\frac{2 \pi}{3}\right) \\
-\sin \theta & \sin \left(\theta-\frac{2 \pi}{3}\right) & \sin \left(\theta+\frac{2 \pi}{3}\right) \\
\frac{1}{\sqrt{2}} & \frac{1}{\sqrt{2}} & \frac{1}{\sqrt{2}}
\end{array}\right] .
$$

The angle between the $d$-axis and the rotor is

$$
\theta=w t+\frac{\pi}{2}
$$

where $w$ is the rated angular frequency in $\mathrm{rad} / \mathrm{s}$. It is easy to find similar expressions for currents and flux linkages:

$$
\begin{aligned}
i_{d q 0} & =P i_{a b c}, \\
\lambda_{d q 0} & =P \lambda_{a b c} .
\end{aligned}
$$

The equivalent circuits of the induction machine are shown in Figure 6. It is convenient to refer all the rotor variables to the stator windings using the turns ratios which is indicated by $\left(v_{d r}^{\prime}, v_{q r}^{\prime}, v_{0 r}^{\prime}\right),\left(i_{d r}^{\prime}, i_{q r}^{\prime}, i_{0 r}^{\prime}\right),\left(\lambda_{d r}^{\prime}, \lambda_{q r}^{\prime}, \lambda_{0 r}^{\prime}\right)$, $L_{l r}^{\prime}, L_{l s}^{\prime}$, and $R_{r}^{\prime}$. Also, $w$ is the synchronous velocity and $w_{r}$ is the rotor velocity. 

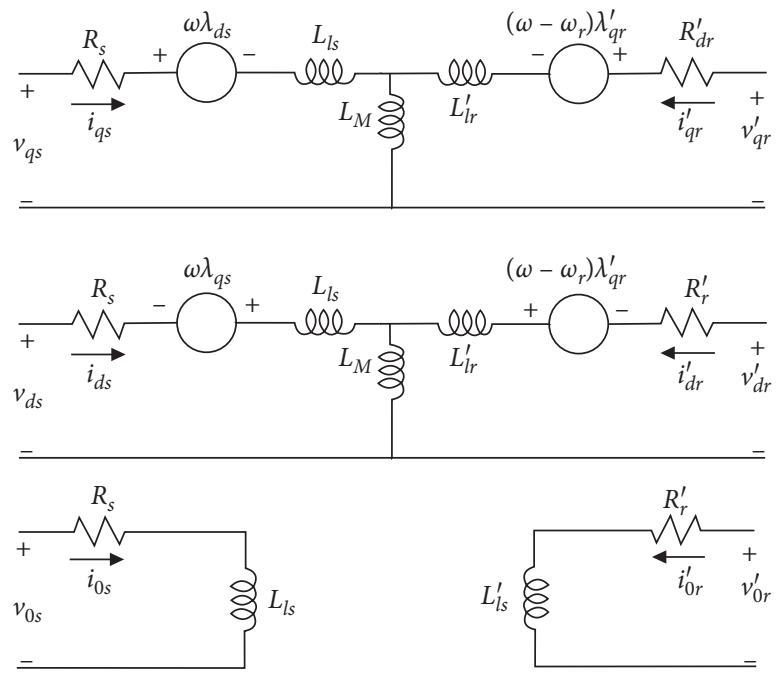

FIgURE 6: Arbitrary reference-frame equivalent circuits for a 3-phase, symmetrical induction machine.

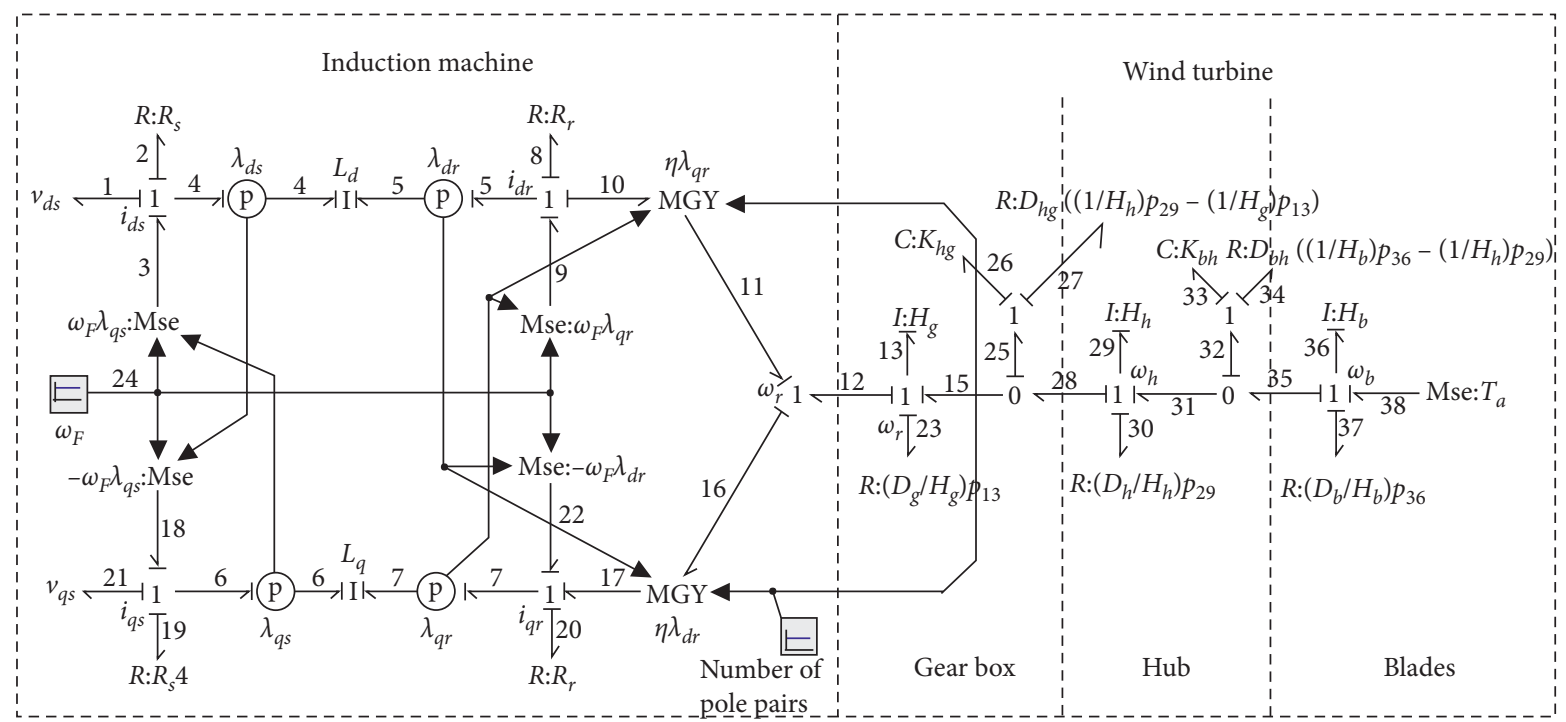

FIGURE 7: Bond graph in an integral causality assignment of the wind turbine.

A bond graph model of an induction machine connected to a wind turbine on the $d-q$ axes is shown in Figure 7. This bond graph contains

(i) $I: L_{d}$ and $I: L_{q}$ are the $I$ fields of the magnetic coupling of the self and mutual inductances of the windings on the $d$-axis and $q$-axis, respectively

(ii) $\left(i_{d r}, \lambda_{d r}\right)$ and $\left(i_{q r}, \lambda_{q r}\right)$ denote the electrical currents and flux linkages for the rotor circuits on the $d$-axis and $q$-axis, respectively

(iii) $R: R_{r}$ and $R: R_{s}$ are the resistances for the rotor and stator circuits, respectively

(iv) $\left(i_{d s}, \lambda_{d s}, v_{d s}\right)$ and $\left(i_{d r}, \lambda_{d r}, v_{d r}\right)$ denote the electrical currents, flux linkages, and voltages for the stator circuits on the $d$-axis and $q$-axis, respectively

(v) $I: H_{g}, I: H_{h}$, and $I: H_{b}$ are the gear box, hub, and blade inertias, respectively

(vi) $R: D_{g} / H_{g}, R: D_{h} / H_{h}$, and $R: D_{b} / H_{b}$ are the gear box, hub, and blade friction coefficient, respectively

(vii) $C: K_{h g}$ and $C: K_{b h}$ denote the stiffness for the shaft and blades, respectively

(viii) $R: D_{h g}\left(\left(p_{29} / H_{h}\right)-\left(p_{13} / H_{g}\right)\right) \quad$ and $R: D_{b h}\left(\left(p_{36} / H_{b}\right)-\left(p_{29} / H_{h}\right)\right)$ are the nonlinear friction coefficients for the gear box and blades, respectively

(ix) $\mathrm{MS}_{e}: T_{a}$ is the plant input representing the aerodynamic torque 
The key vectors of the bond graph for the dissipation field and input are

$$
\begin{aligned}
& D_{\text {in }}^{l}=\left[\begin{array}{c}
f_{2} \\
f_{8} \\
f_{19} \\
f_{20}
\end{array}\right] \text {, } \\
& D_{\text {out }}^{l}=\left[\begin{array}{c}
e_{2} \\
e_{8} \\
e_{19} \\
e_{20}
\end{array}\right] \text {, } \\
& D_{\text {in }}^{n l}=\left[\begin{array}{l}
f_{23} \\
f_{27} \\
f_{30} \\
f_{34} \\
f_{37}
\end{array}\right] \text {, } \\
& D_{\text {out }}^{n l}=\left[\begin{array}{c}
e_{23} \\
e_{27} \\
e_{30} \\
e_{34} \\
e_{37}
\end{array}\right] \text {, } \\
& u=\left[\begin{array}{c}
e_{1} \\
e_{21} \\
e_{38} \\
f_{24}
\end{array}\right] \text {, }
\end{aligned}
$$

for the slow dynamics

$$
\begin{aligned}
& x_{1}=\left[\begin{array}{l}
p_{13} \\
q_{26} \\
p_{29} \\
q_{33} \\
p_{36}
\end{array}\right], \\
& \dot{x}_{1}=\left[\begin{array}{l}
e_{13} \\
f_{26} \\
e_{29} \\
f_{33} \\
e_{36}
\end{array}\right], \\
& z_{1}=\left[\begin{array}{l}
f_{13} \\
e_{26} \\
f_{29} \\
e_{33} \\
f_{36}
\end{array}\right],
\end{aligned}
$$

and for the fast dynamics

$$
\begin{gathered}
x_{2}=\left[\begin{array}{l}
p_{4} \\
p_{5} \\
p_{6} \\
p_{7}
\end{array}\right], \\
\dot{x}_{2}=\left[\begin{array}{l}
e_{4} \\
e_{5} \\
e_{6} \\
e_{7}
\end{array}\right], \\
z_{2}=\left[\begin{array}{l}
f_{4} \\
f_{5} \\
f_{6} \\
f_{7}
\end{array}\right],
\end{gathered}
$$

where $f_{24}=\omega_{F}$ is the synchronous velocity.

The constitutive relations of the fields are

$$
\begin{aligned}
& L=\operatorname{diag}\left\{R_{s}, R_{r}, R_{s}, R_{r}\right\}, \\
& \phi_{L}=\operatorname{diag}\left\{\frac{D_{g}}{H_{g}} p_{13}, D_{h g}\left(\frac{1}{H_{h}} p_{29}-\frac{1}{H_{g}} p_{13}\right)\right. \text {, } \\
& \left.D_{b h}\left(\frac{1}{H_{b}} p_{36}-\frac{1}{H_{h}} p_{29}\right), \frac{D_{b}}{H_{b}} p_{36}\right\} \text {, } \\
& F_{1}^{-1}=\operatorname{diag}\left\{H_{g}, K_{h g}, H_{h}, K_{b h}, H_{b}\right\} \text {, } \\
& F_{2}^{-1}=\left[\begin{array}{cccc}
L_{s} & L_{m} & 0 & 0 \\
L_{m} & L_{r} & 0 & 0 \\
0 & 0 & L_{s} & L_{m} \\
0 & 0 & L_{m} & L_{r}
\end{array}\right] .
\end{aligned}
$$

The junction structure of the bond graph is given by

$$
\left[\begin{array}{c}
\dot{x}_{1} \\
\dot{x}_{2} \\
D_{\text {in }}^{l} \\
D_{\text {in }}^{n l}
\end{array}\right]=\left[\begin{array}{lll}
S_{11}(x) & S_{12}(x) & S_{13}(x) \\
S_{21}(x) & S_{22}(x) & S_{23}(x)
\end{array}\right]\left[\begin{array}{c}
z_{1} \\
z_{2} \\
D_{\text {out }}^{l} \\
D_{\text {out }}^{n l} \\
u
\end{array}\right] .
$$

The developed junction structure is defined in Appendix C.

The mathematical model of the complete system is determined. Firstly, for the slow dynamics, from (13) to (17) and (20) with (75) to (C.1), the matrix $A_{11}(x)$ is expressed by 


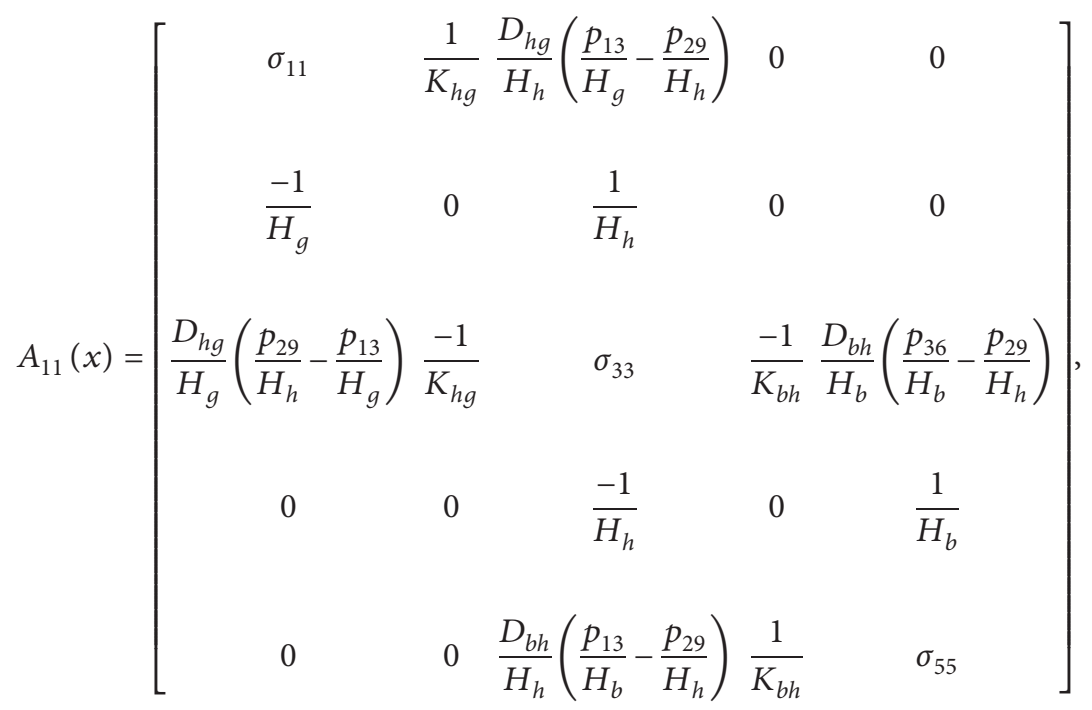

where

$$
\begin{aligned}
& \sigma_{11}=\frac{1}{H_{g}}\left[D_{h g}\left(\frac{p_{13}}{H_{g}}-\frac{p_{29}}{H_{h}}\right)-\frac{D_{g}}{H_{g}} p_{13}\right], \\
& \sigma_{33}=\frac{-1}{H_{h}}\left[D_{b h}\left(\frac{p_{36}}{H_{b}}-\frac{p_{29}}{H_{h}}\right)-D_{h g}\left(\frac{p_{13}}{H_{g}}-\frac{p_{29}}{H_{h}}\right)+\frac{D_{h}}{H_{h}} p_{29}\right], \\
& \sigma_{55}=\frac{-1}{H_{b}}\left[D_{b h}\left(\frac{p_{36}}{H_{b}}-\frac{p_{29}}{H_{h}}\right)+\frac{D_{b}}{H_{b}} p_{36}\right],
\end{aligned}
$$

the relationships between the slow and fast dynamics denoted by $A_{12}(x)$ is obtained from (13) to (17) and (21) with (75) to (C.1):

$$
A_{12}(x)=\frac{1}{\Delta}\left[\begin{array}{cccc}
-\eta L_{m} p_{7} & \eta L_{s} p_{7} & \eta L_{m} p_{5} & -\eta L_{s} p_{5} \\
0 & 0 & 0 & 0 \\
0 & 0 & 0 & 0 \\
0 & 0 & 0 & 0 \\
0 & 0 & 0 & 0
\end{array}\right] \text {, }
$$

where $\Delta=L_{r} L_{s}-L_{m}^{2}$.

From (16), (24), (75), and (C.1), the matrix $B_{1}$ is

$$
B_{1}=\left[\begin{array}{llll}
0 & 0 & 0 & 0 \\
0 & 0 & 0 & 0 \\
0 & 0 & 0 & 0 \\
0 & 0 & 0 & 0 \\
0 & 0 & 1 & 0
\end{array}\right] .
$$

The matrices of the state variables representation for the fast dynamics are obtained. From (13) to (16) and (22) with (75) to (C.1), the matrix $A_{21}(x)$ is given by

$$
A_{21}(x)=\left[\begin{array}{lllll}
\frac{-\eta L_{m}}{H_{g}} p_{7} & 0 & 0 & 0 & 0 \\
\frac{-\eta L_{r}}{H_{g}} p_{7} & 0 & 0 & 0 & 0 \\
\frac{\eta L_{m}}{H_{g}} p_{5} & 0 & 0 & 0 & 0 \\
\frac{\eta L_{r}}{H_{g}} p_{5} & 0 & 0 & 0 & 0
\end{array}\right] .
$$

By substituting (75) to (C.1) into (23) with (13) to (16), the matrix $A_{22}(x)$ is 


$$
A_{22}(x)=\frac{1}{\Delta}\left[\begin{array}{cccc}
L_{m}^{2} R_{r}-L_{r} L_{s} R_{s} & L_{m} L_{s} R_{s}-L_{m} L_{s} R_{r} & 0 & 0 \\
L_{m} L_{r} R_{r}-L_{m} L_{r} R_{s} & L_{m}^{2} R_{s}-L_{r} L_{s} R_{r} & 0 & 0 \\
0 & 0 & L_{m}^{2} R_{r}-L_{r} L_{s} R_{s} & L_{m} L_{s} R_{s}-L_{m} L_{s} R_{r} \\
0 & 0 & L_{m} L_{r} R_{r}-L_{m} L_{r} R_{s} & L_{m}^{2} R_{s}-L_{r} L_{s} R_{r}
\end{array}\right] .
$$

From (15), (16), and (25) with (75) to (C.1), the matrix $B_{2}(x)$ is

$$
B_{2}(x)=\left[\begin{array}{cccc}
-L_{s} & 0 & 0 & L_{s} p_{6}+L_{m} p_{7} \\
-L_{m} & 0 & 0 & L_{s} p_{6}+L_{m} p_{7} \\
0 & -L_{s} & 0 & -\left(L_{s} p_{4}+L_{m} p_{5}\right) \\
0 & -L_{m} & 0 & -\left(L_{s} p_{4}+L_{r} p_{5}\right)
\end{array}\right] .
$$

It can be seen that the system order is $n+m=9$, where the slow dynamics are $n=5$ and the fast dynamics are $m=4$.

\section{A Quasi-Steady State Model of the Wind Turbine in a Bond Graph Approach}

In this section, the main contribution of this paper is described. In order to get the quasi-steady state model of a nonlinear system with singular perturbations, the traditional approach given in Section 2 requires many mathematical steps. Hence, the corresponding bond graph of Figure 7 describes the mathematical model of the case study of this paper. This bond graph contains all the storage elements of both dynamics in integral causality assignment. For the storage elements with fast dynamics, a derivative causality is assigned. For the storage elements with slow dynamics, an integral causality is maintained and a quasi-steady state model of a singularly perturbed nonlinear system can be obtained. The key vectors of the storage elements and inputs for this SPNBG are the same with respect to NBGI. Also, the new key vectors of the bond graph of Figure 8 for the linear dissipation elements are

$$
D_{\text {in }}^{h l}=\left[\begin{array}{c}
e_{2} \\
e_{8} \\
e_{19} \\
e_{20}
\end{array}\right]
$$

$$
D_{\text {out }}^{h l}=\left[\begin{array}{c}
f_{2} \\
f_{8} \\
f_{19} \\
f_{20}
\end{array}\right] \text {, }
$$

and the constitutive relation is

$$
L^{h}=\operatorname{diag}\left\{\frac{1}{R_{s}}, \frac{1}{R_{r}}, \frac{1}{R_{s}}, \frac{1}{R_{r}}\right\} .
$$

From (38), the junction structure of the bond graph of Figure 8 is described in Appendix D.

From (38)-(41) and (75) to (C.1) with (87) and (D.1), the real roots of the algebraic equation can be obtained by

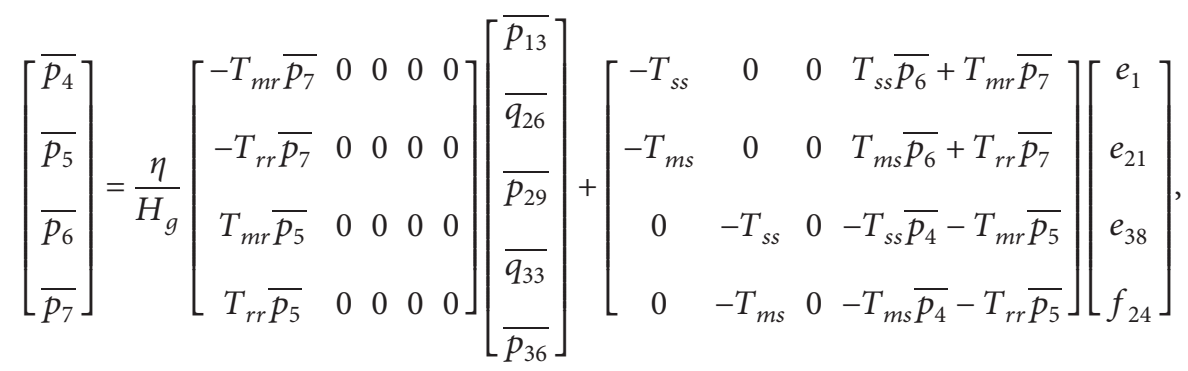

where

$$
\begin{gathered}
T_{s s}=\frac{L_{s}}{R_{s}}, \\
T_{r r}=\frac{L_{r}}{R_{r}}, \\
T_{m s}=\frac{L_{m}}{R_{s}}, \\
T_{m r}=\frac{L_{m}}{R_{r}} .
\end{gathered}
$$

By substituting (77)-(79) with (86) and (87) into (42) with (43) and (45), the quasi-steady state model is defined by 


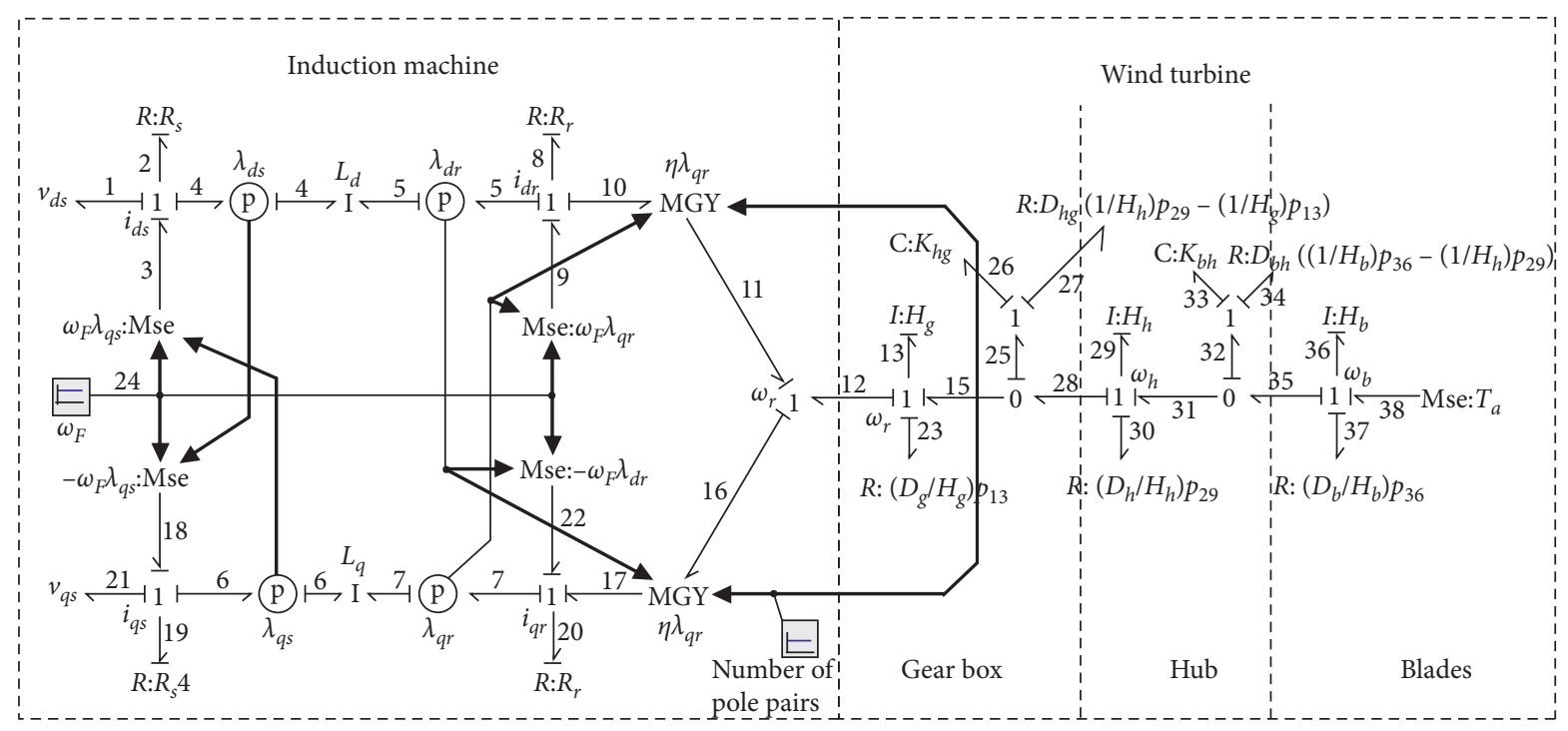

Figure 8: Bond graph with a derivative causality assignment for the fast dynamics of the wind turbine.

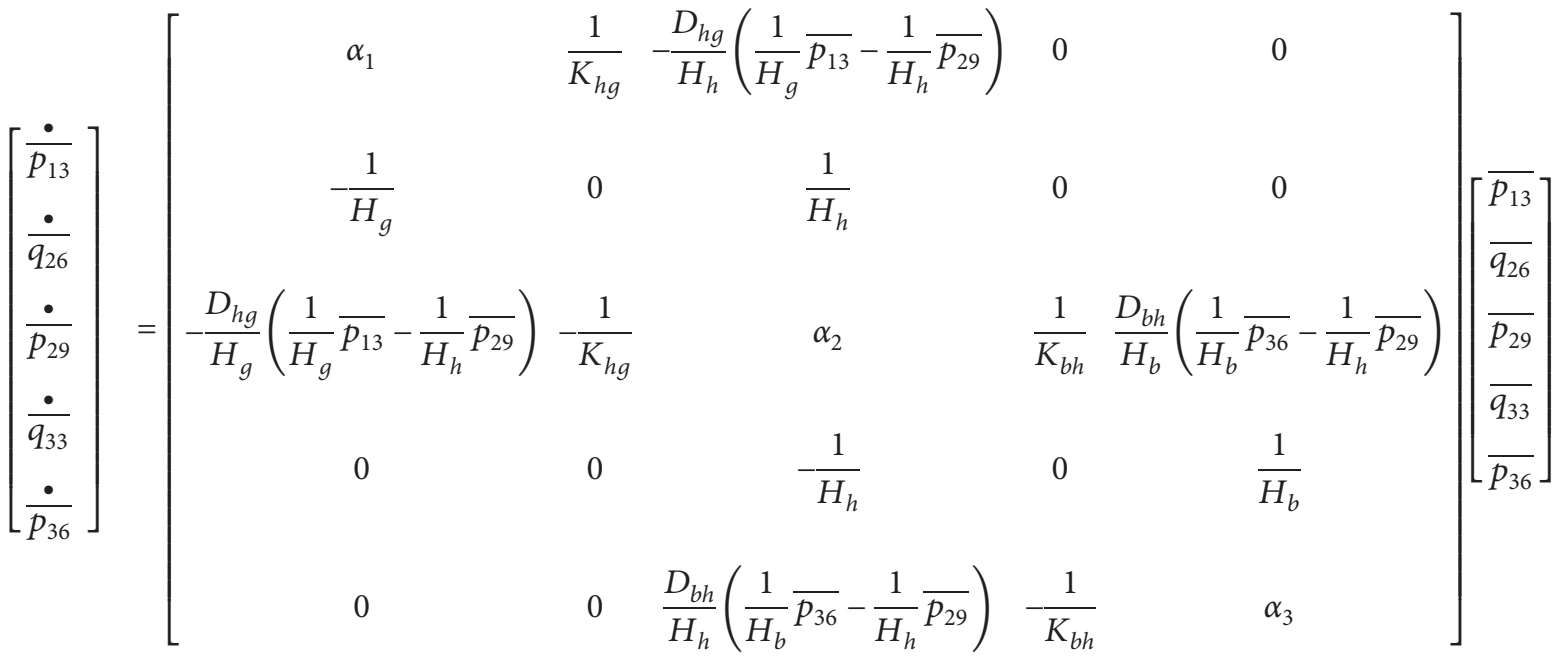

$$
\begin{aligned}
& +\left[\begin{array}{cccc}
0 & 0 & 0 & \frac{\eta}{R_{r}}\left({\overline{p_{5}}}^{2}+{\overline{p_{7}}}^{2}\right) \\
0 & 0 & 0 & 0 \\
0 & 0 & 0 & 0 \\
0 & 0 & 0 & 0 \\
0 & 0 & 1 & 0
\end{array}\right]\left[\begin{array}{c}
e_{1} \\
e_{21} \\
e_{38} \\
f_{24}
\end{array}\right],
\end{aligned}
$$

where $\alpha_{1}, \alpha_{2}$, and $\alpha_{3}$ are

$$
\begin{aligned}
& \alpha_{1}=-\frac{1}{H_{g}}\left[\frac{\eta^{2}}{R_{r}}\left({\overline{p_{5}}}^{2}+{\overline{p_{7}}}^{2}\right)-D_{h g}\left(\frac{1}{H_{g}} \overline{p_{13}}-\frac{1}{H_{h}} \overline{p_{29}}\right)+\frac{D_{g}}{H_{g}} \overline{p_{13}}\right], \\
& \alpha_{2}=-\frac{1}{H_{h}}\left[D_{b h}\left(\frac{1}{H_{b}} \overline{p_{36}}-\frac{1}{H_{h}} \overline{p_{29}}\right)-D_{h g}\left(\frac{1}{H_{g}} \overline{p_{13}}-\frac{1}{H_{h}} \overline{p_{29}}\right)+\frac{D_{h}}{H_{h}} \overline{p_{29}}\right], \\
& \alpha_{3}=-\frac{1}{H_{b}}\left[D_{b h}\left(\frac{1}{H_{b}} \overline{p_{36}}-\frac{1}{H_{h}} \overline{p_{29}}\right)+\frac{D_{b}}{H_{b}} \overline{p_{36}}\right] .
\end{aligned}
$$



have

By solving the first, second, and fourth lines of (88), we

$$
\begin{aligned}
\overline{p_{5}}=\frac{K_{2} K_{3}+K_{5} K_{4}}{K_{5} K_{6}-K_{1} K_{3}}=K_{7}, \\
\overline{p_{4}}=\frac{K_{7} K_{1}+K_{2}}{K_{5}}, \\
K_{1}=\frac{\eta T_{m r}}{H_{g}} \overline{p_{13}}\left[-\frac{\eta T_{r r}}{H_{g}} \overline{p_{13}}+\left(2 T_{r r}+T_{s s}\right) f_{24}\right]-T_{m r}\left(T_{s s}+T_{r r}\right) f_{24}^{2}, \\
K_{2}=T_{m r} T_{m s} e_{21}\left(\frac{\eta}{H_{g}} \overline{p_{13}}-f_{24}\right)-T_{s s} e_{1}-T_{s s}^{2} f_{24} e_{21}, \\
K_{3}=T_{r r} T_{m s} f_{24}\left(\frac{\eta}{H_{g}} \overline{p_{13}}-f_{24}\right)-T_{s s} T_{m s} f_{24}^{2}, \\
K_{4}=T_{r r} T_{m s} e_{21}\left(\frac{\eta}{H_{g}} \overline{p_{13}}-f_{24}\right)-T_{m s} e_{1}-T_{s s} T_{m s} f_{24} e_{21}, \\
K_{5}=1+T_{m r} T_{m s} f_{24}\left(-\frac{\eta}{H_{g}} \overline{p_{13}}+f_{24}\right)+T_{s s}^{2} f_{24}^{2}, \\
K_{6}=1+\frac{\eta}{H_{g}} \overline{p_{13}}\left[\frac{\eta T_{r r}^{2}}{H_{g}} \overline{p_{13}}-\left(2 T_{r r}^{2}+T_{m r} T_{m s}\right) f_{24}\right]+\left(T_{m r} T_{m s}+T_{r r}^{2}\right) f_{24}^{2} .
\end{aligned}
$$

By substituting (93) to (96) into (88) and (90), the quasisteady state model is defined by

$$
\begin{aligned}
\dot{\overline{p_{13}}}= & -\frac{1}{H_{g}}\left[\frac{\eta^{2}}{R_{r}}\left({\overline{p_{5}}}^{2}+{\overline{p_{7}}}^{2}\right)-D_{h g}\left(\frac{1}{H_{g}} \overline{p_{13}}-\frac{1}{H_{h}} \overline{p_{29}}\right)+\frac{D_{g}}{H_{g}} \overline{p_{13}}\right] \overline{p_{13}}+\frac{1}{K_{h g}} \overline{q_{26}} \\
& -\frac{D_{h g}}{H_{h}}\left(\frac{1}{H_{g}} \overline{p_{13}}-\frac{1}{H_{h}} \overline{p_{29}}\right) \overline{p_{29}}+\frac{\eta}{R_{r}}\left({\overline{p_{5}}}^{2}+{\overline{p_{7}}}^{2}\right) f_{24}, \\
\dot{\overline{q_{26}}}= & -\frac{1}{H_{g}} \overline{p_{13}}+\frac{1}{H_{h}} \overline{p_{29}}, \\
\dot{p_{29}}= & -\frac{D_{h g}}{H_{g}}\left(\frac{1}{H_{g}} \overline{p_{13}}-\frac{1}{H_{h}} \overline{p_{29}}\right) \overline{p_{13}}-\frac{1}{K_{h g}} \overline{q_{26}}- \\
& -\frac{1}{H_{h}}\left[D_{b h}\left(\frac{1}{H_{b}} \overline{p_{36}}-\frac{1}{H_{h}} \overline{p_{29}}\right)-D_{h g}\left(\frac{1}{H_{g}} \overline{p_{13}}-\frac{1}{H_{h}} \overline{p_{29}}\right)+\frac{D_{h}}{H_{h}} \overline{p_{29}}\right] \overline{p_{29}}+ \\
& +\frac{1}{K_{b h}} \overline{q_{33}}+\frac{D_{b h}}{H_{b}}\left(\frac{1}{H_{b}} \overline{p_{36}}-\frac{1}{H_{h}} \overline{p_{29}}\right) \overline{p_{36}}, \\
\dot{\overline{q_{33}}=} & -\frac{1}{H_{h}} \overline{p_{29}}+\frac{1}{H_{b}} \overline{p_{36}},
\end{aligned}
$$




$$
\dot{\bullet}=\frac{D_{b h}}{H_{h}}\left(\frac{1}{\hat{p}_{b}} \overline{p_{36}}-\frac{1}{H_{h}} \overline{p_{29}}\right) \overline{p_{29}}-\frac{1}{K_{b h}} \overline{q_{33}}-\frac{1}{H_{b}}\left[D_{b h}\left(\frac{1}{H_{b}} \overline{p_{36}}-\frac{1}{H_{h}} \overline{p_{29}}\right)+\frac{D_{b}}{H_{b}} \overline{p_{36}}\right] \overline{p_{36}}+e_{38} .
$$

Section 7 describes the performance of the state variables of the wind turbine connected to an induction machine.

\section{Simulation Results}

In order to verify the quasi-steady state model of the complete system, the simulations results are obtained. Hence, the parameters of the induction generator were obtained from the literature [30]. These parameters are $H_{g}=0.089 \mathrm{kgm}^{2}, R_{s}=0.435 \Omega, R_{r}=0.816 \Omega, L_{s}=0.0713 \mathrm{H}$, $L_{r}=0.0713 \mathrm{H}, \quad L_{m}=0.0693 \mathrm{H}, \quad \eta=2$ pairs of poles, $V_{\text {nom }}=220 \mathrm{~V}$, and $\omega_{F}=377 \mathrm{rad} / \mathrm{s}$. And, for the mechanical model, $H_{b}=0.05 \mathrm{kgm}^{2}, H_{h}=0.03 \mathrm{kgm}^{2}, D_{b h}=0.4 \mathrm{~N}-\mathrm{m}-\mathrm{s}$, $D_{h g}=0.4 \mathrm{~N}-\mathrm{m}-\mathrm{s}, D_{b}=0.4 \mathrm{~N}-\mathrm{m}-\mathrm{s}, D_{h}=0.3 \mathrm{~N}-\mathrm{m}-\mathrm{s}, D_{g}=0.1 \mathrm{~N}-$ $\mathrm{m}-\mathrm{s}, \quad K_{b h}=1 e^{-3} N-\mathrm{m} / \mathrm{rad}, \quad K_{h g}=1 e^{-3} \mathrm{Nm} / \mathrm{rad}, \quad$ and $T_{a}=100 \mathrm{Nm}$.

$$
\operatorname{Re\lambda }\left\{\left[\begin{array}{cc}
\frac{L_{m}^{2} R_{r}-L_{r} L_{s} R_{s}}{L_{r} L_{s}-L_{m}^{2}} & \frac{L_{m} L_{s} R_{s}-L_{m} L_{s} R_{r}}{L_{r} L_{s}-L_{m}^{2}} \\
\frac{L_{m} L_{r} R_{r}-L_{m} L_{r} R_{s}}{L_{r} L_{s}-L_{m}^{2}} & \frac{L_{m}^{2} R_{s}-L_{r} L_{s} R_{r}}{L_{r} L_{s}-L_{m}^{2}} \\
-L_{s} f_{24} & -L_{m} f_{24} \\
-L_{m} f_{24} & -L_{r} f_{24}
\end{array}\right.\right.
$$

and substituting the numerical parameters of the system into (103),

$$
\operatorname{Re\lambda }\left\{\left[\begin{array}{cccc}
6.0719 & -6.6947 & 26.8801 & 26.1261 \\
6.6947 & -7.3229 & 26.1261 & 26.8801 \\
-26.8801 & -26.1261 & 6.07192 & -6.6947 \\
-26.1261 & -26.8801 & 6.6947 & -7.3229
\end{array}\right]\right\} .
$$

Then, the eigenvalues are

$$
\begin{aligned}
& \lambda_{1}=-0.6255+j 53.0055, \\
& \lambda_{2}=-0.6255-j 53.0055, \\
& \lambda_{3}=-0.6255+j 0.7547, \\
& \lambda_{4}=-0.6255-j 0.7547,
\end{aligned}
$$

and (12) is satisfied, and we can conclude that this singularly perturbed system has internal stability.
It is assumed that the machine is connected to a bus whose voltage and frequency remain constant, at the rated values. This is commonly known as an infinite bus. It is necessary to verify (12) with (36) and (37):

$$
\operatorname{Re}\left\{\frac{\partial\left[A_{21}(x) x_{1}(t)\right]}{\partial x_{2}}+\frac{\partial\left[A_{22}(x) x_{2}(t)\right]}{\partial x_{2}}+\frac{\partial\left[B_{2}(x) u(t)\right]}{\partial x_{2}}\right\} \leq 0 .
$$

The first partial derivative is $\partial\left[A_{21}(x) x_{1}\right] / \partial x 2=0$, according to (12), then (101) is reduced to

$$
\operatorname{Re}\left\{\frac{\partial\left[A_{22}(x) x_{2}(t)\right]}{\partial x_{2}}+\frac{\partial\left[B_{2}(x) u(t)\right]}{\partial x_{2}}\right\} \leq 0 \text {. }
$$

By solving (102),

$$
\left.\left.\begin{array}{cc}
L_{s} f_{24} & L_{m} f_{24} \\
L_{m} f_{24} & L_{r} f_{24} \\
\frac{L_{m}^{2} R_{r}-L_{r} L_{s} R_{s}}{L_{r} L_{s}-L_{m}^{2}} & \frac{L_{m} L_{s} R_{s}-L_{m} L_{s} R_{r}}{L_{r} L_{s}-L_{m}^{2}} \\
\frac{L_{m} L_{r} R_{r}-L_{m} L_{r} R_{s}}{L_{r} L_{s}-L_{m}^{2}} & \frac{L_{m}^{2} R_{s}-L_{r} L_{s} R_{r}}{L_{r} L_{s}-L_{m}^{2}}
\end{array}\right]\right\} \leq 0,
$$

It is instructive to observe the variables for the slow and fast dynamics considering the complete and reduced models. Figures 9-11 show the angular momentum of the hub, blades, and gear box for the exact $\left(p_{29}, p_{36}, p_{13}\right)$ and reduced $\left(\overline{p_{29}}, \overline{p_{36}}, \overline{p_{13}}\right)$ models, respectively.

The oscillations of the angles for the exact $\left(q_{26}, q_{33}\right)$ and reduced $\left(\overline{q_{26}}, \overline{q_{33}}\right)$ models, respectively, are shown in Figures 12 and 13, where $q_{26}$ is the angle between the generator and the hub and $q_{33}$ the angle related to the hub and the blades of the wind turbine.

In order to prove that this system has singular perturbations, Figure 14 illustrates the electrical current on $d$-axis $\left(f_{5}\right)$ that represents a fast dynamic, and the slow dynamic into this figure corresponds the angular velocity of the rotor $\left(f_{13}\right)$.

Note that the proposed methodology allows to obtain the quasi-steady state model by assigning a derivative causality to the storage elements for the fast dynamics and an integral causality assignment to the storage elements for the slow 


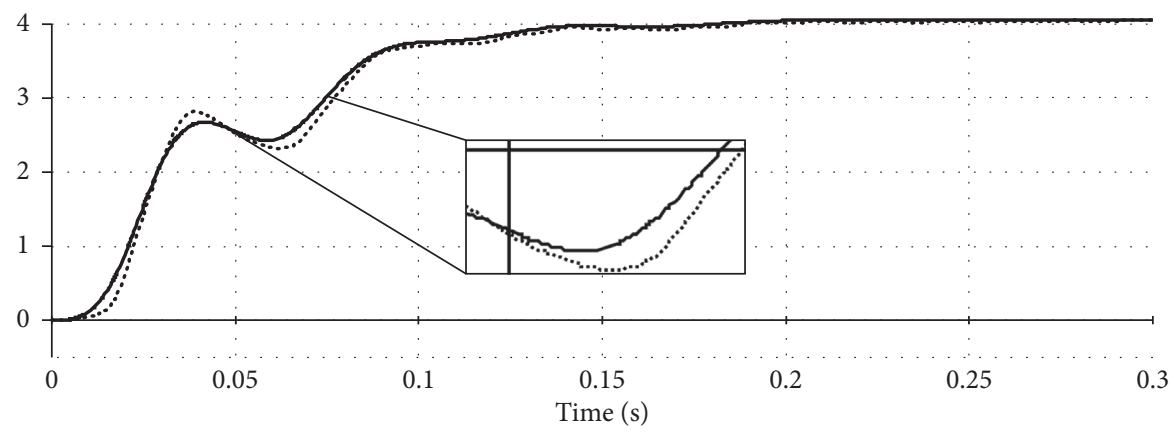

$\overline{p_{29}}(\mathrm{~N}-\mathrm{m}-\mathrm{s})$

…. $p_{29}(\mathrm{~N}-\mathrm{m}-\mathrm{s})$

Figure 9: Performance of the slow variable $p_{29}$ and the quasi-steady state model $\overline{p_{29}}$.

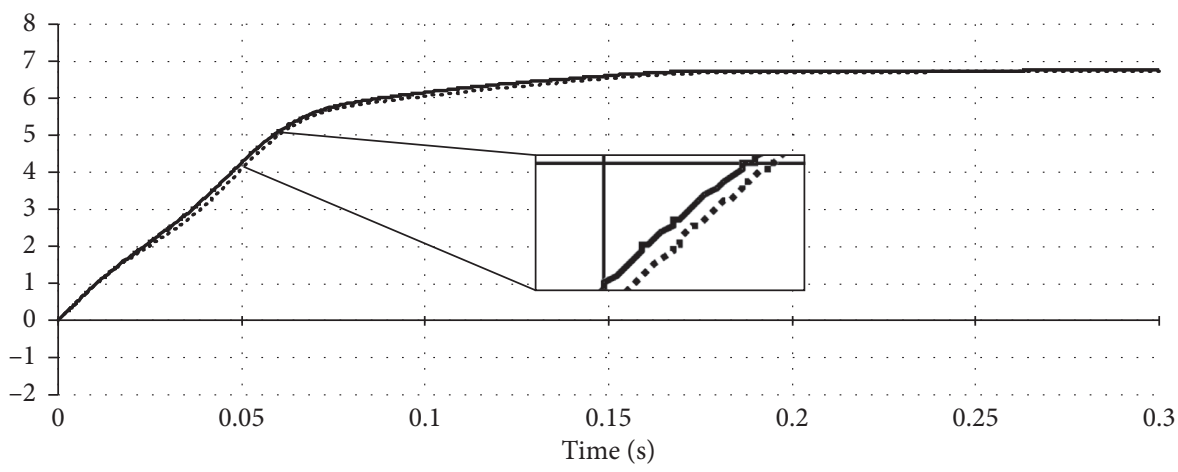

$$
\begin{aligned}
& -\overline{p_{36}}(\mathrm{~N}-\mathrm{m}-\mathrm{s}) \\
& \cdots \cdots p_{36}(\mathrm{~N}-\mathrm{m}-\mathrm{s})
\end{aligned}
$$

Figure 10: Performance of the slow variable $p_{36}$ and the quasi-steady state model $\overline{p_{36}}$.

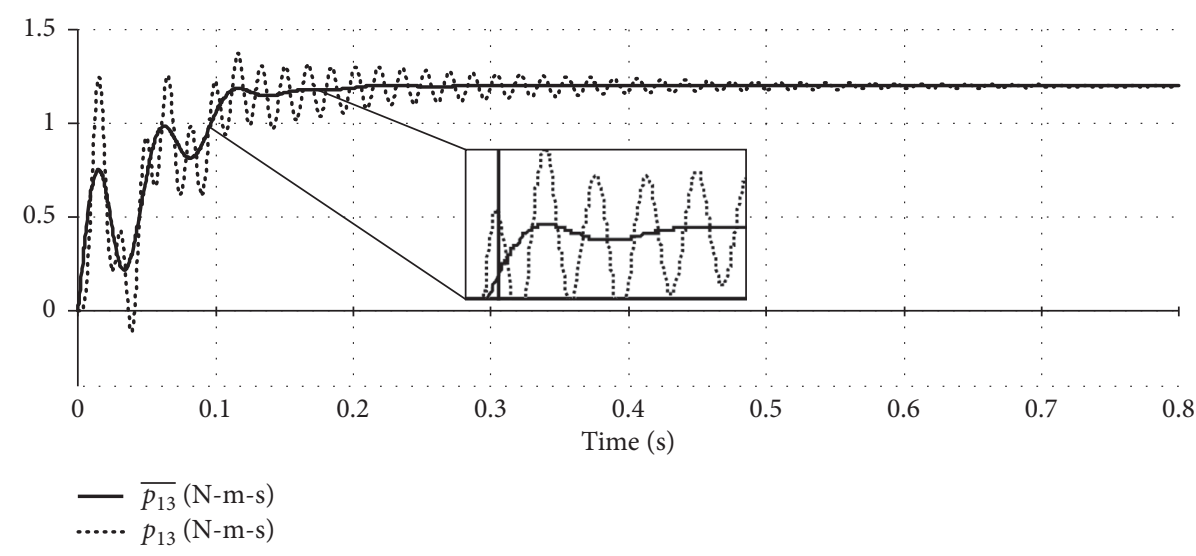

FIgURE 11: Performance of the slow variable and its respective quasi-steady responses: $p_{13}$ and. $\overline{p_{13}}$.

dynamics of a nonlinear system with singular perturbations. The previous simulation results were obtained considering a constant torque $T_{a}$. Now, a variable torque is supplied to the bond graph model of Figure 7. This torque is obtained from a real wind speed data which is shown in Figure 13, taken from the measurement of a wind turbine installed in the University of Michoacan in Mexico.
The torque $\mathrm{Ta}$ is determined by

$$
T a=K V_{\text {wind }}^{2}
$$

where $V_{\text {wind }}$ is the wind speed and $K$ is the constant to transfer wind speed to a torque. The dynamic behavior of the exact and approximate variables of the system is shown in Figures 14-25. 


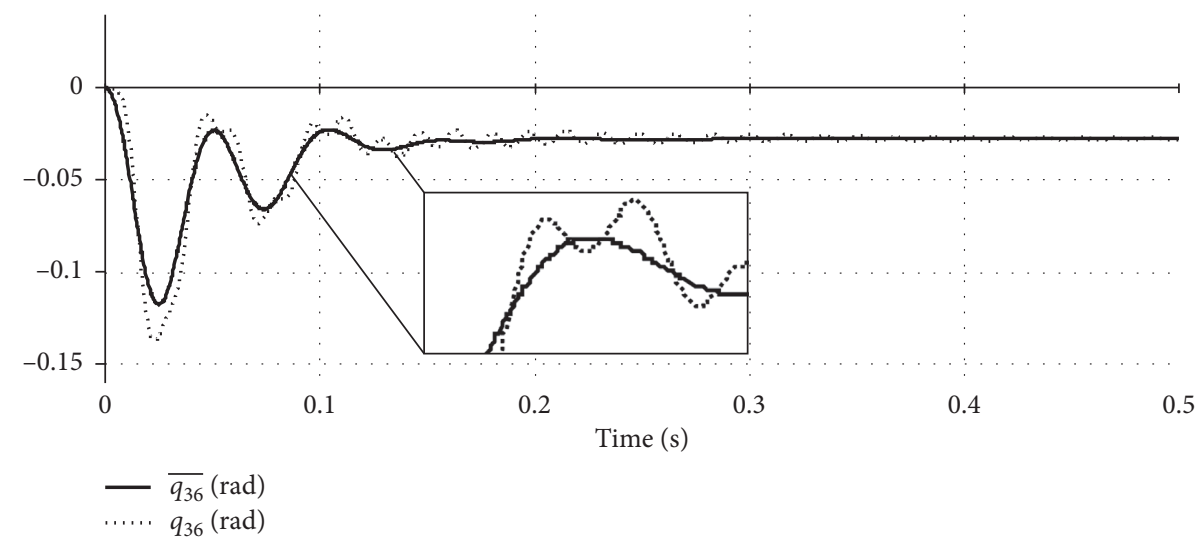

FIgURe 12: Performance of the slow variable $q_{26}$ and the quasi-steady state response $\overline{q_{26}}$.

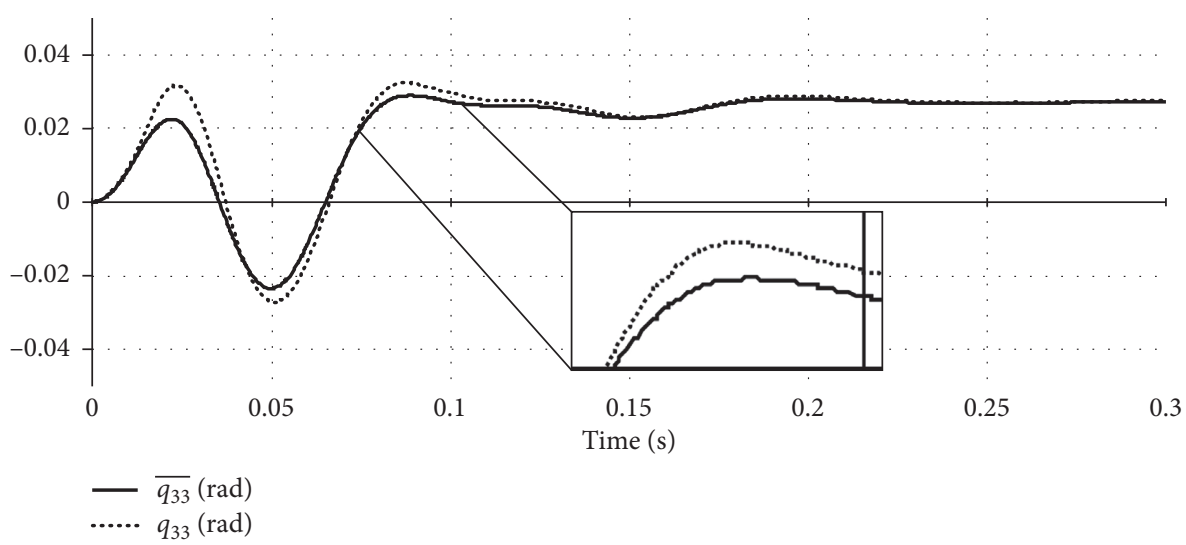

FIGURE 13: Performance of the slow variable $q_{33}$ and its respective quasi-steady state response $\overline{q_{33}}$.

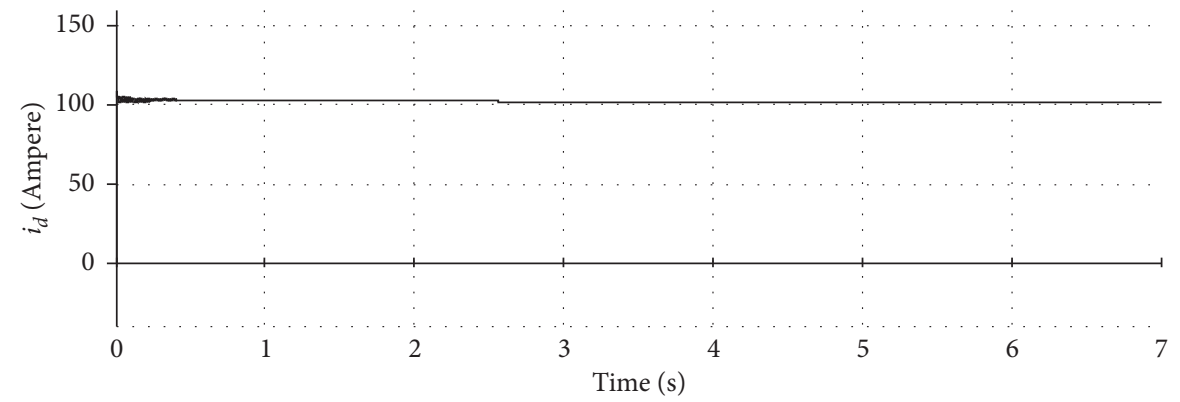

(a)

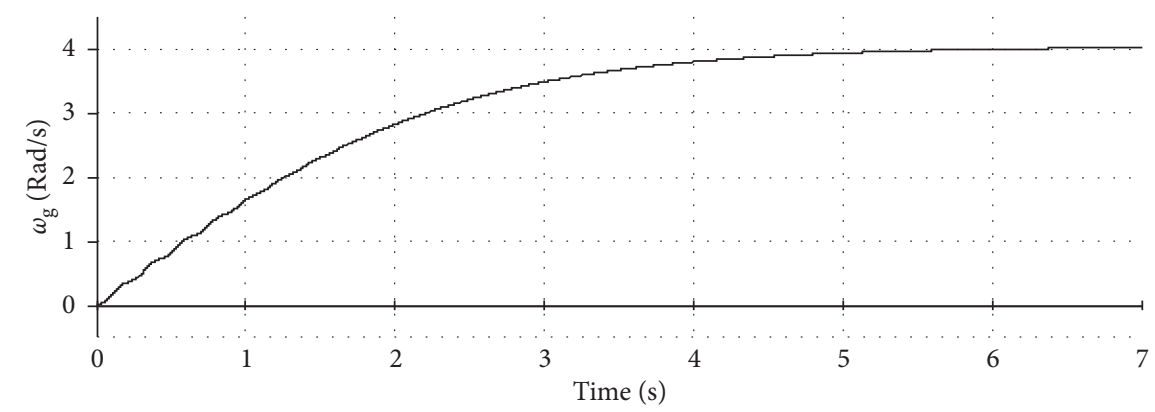

(b)

FIgure 14: (a) Performance of the current on $d$-axis, $i_{d}$ (fast variable). (b) The angular velocity of the rotor, $\omega_{\mathrm{g}}$. 


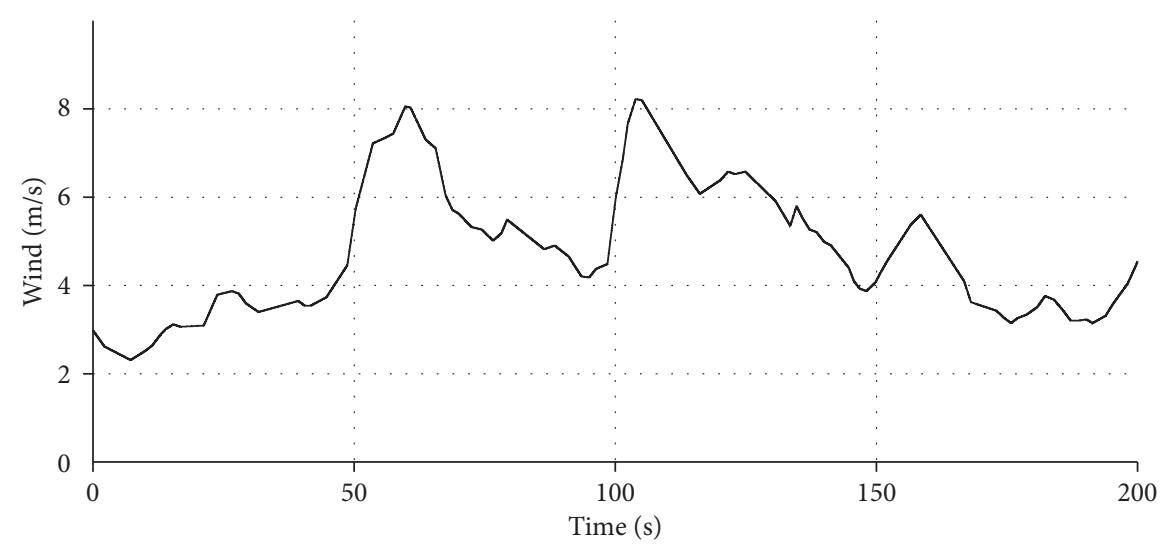

FIgURE 15: Real wind speed data.

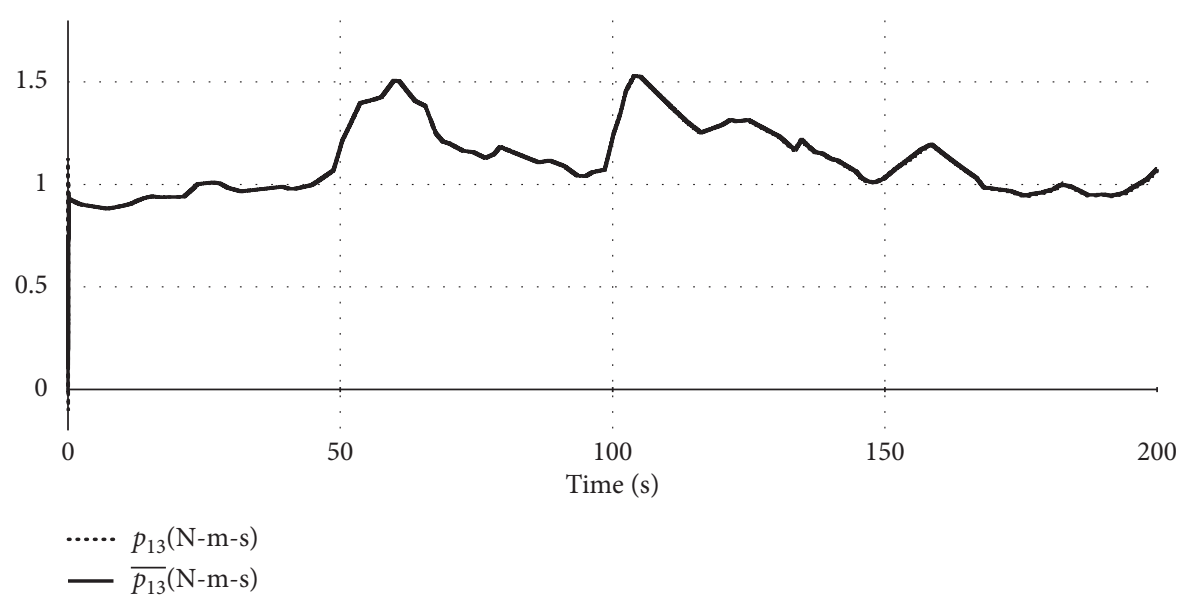

Figure 16: Full time simulation of the slow variable $p_{13}$ and the quasi-steady state model $\overline{p_{13}}$.

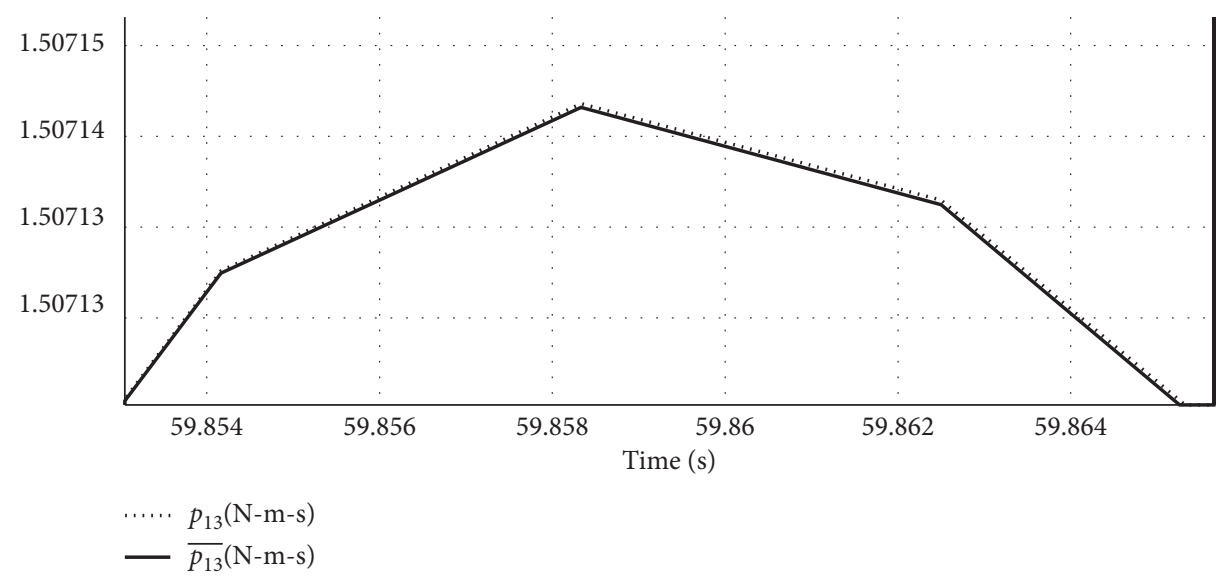

FIgURE 17: Close up of Figure 16. 


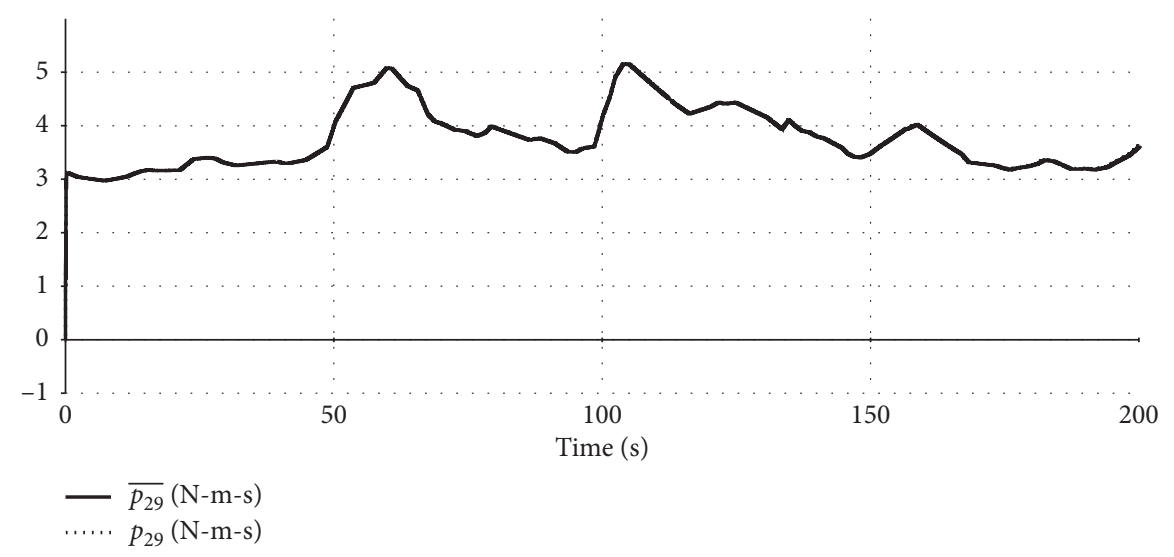

Figure 18: Full-time simulation of the slow variable $p_{29}$ and the quasi-steady state model $\overline{p_{29}}$.

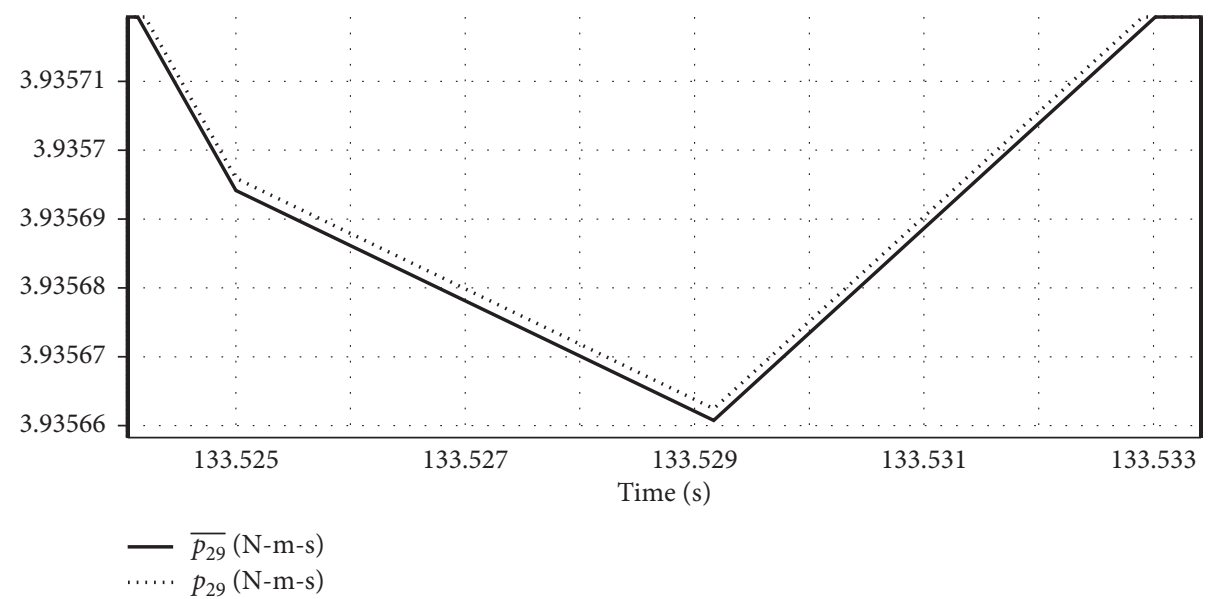

FIgURE 19: Close up of Figure 18.

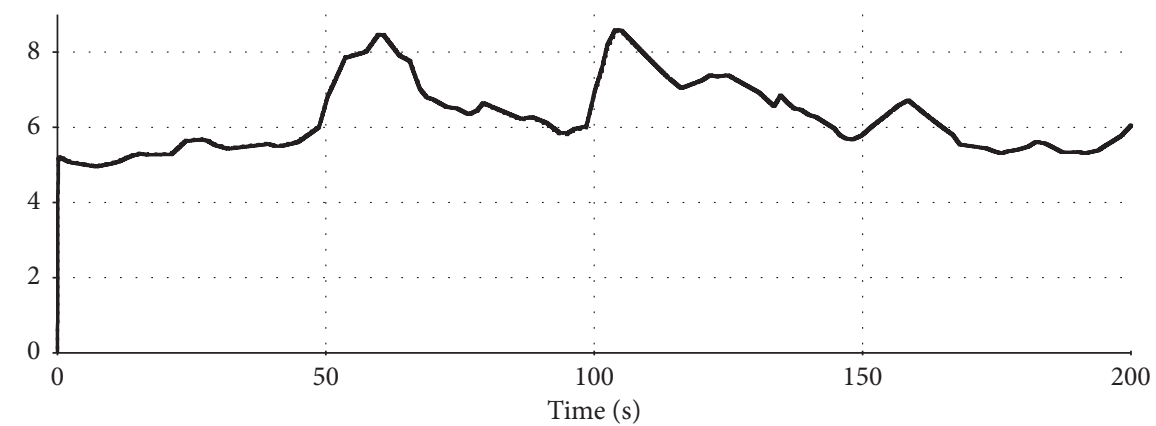

$-\overline{p_{36}}(\mathrm{~N}-\mathrm{m}-\mathrm{s})$
$\cdots \cdots$
$p_{36}(\mathrm{~N}-\mathrm{m}-\mathrm{s})$

FIGURE 20: Full-time simulation of the slow variable $p_{36}$ and the quasi-steady state model $\overline{p_{36}}$. 


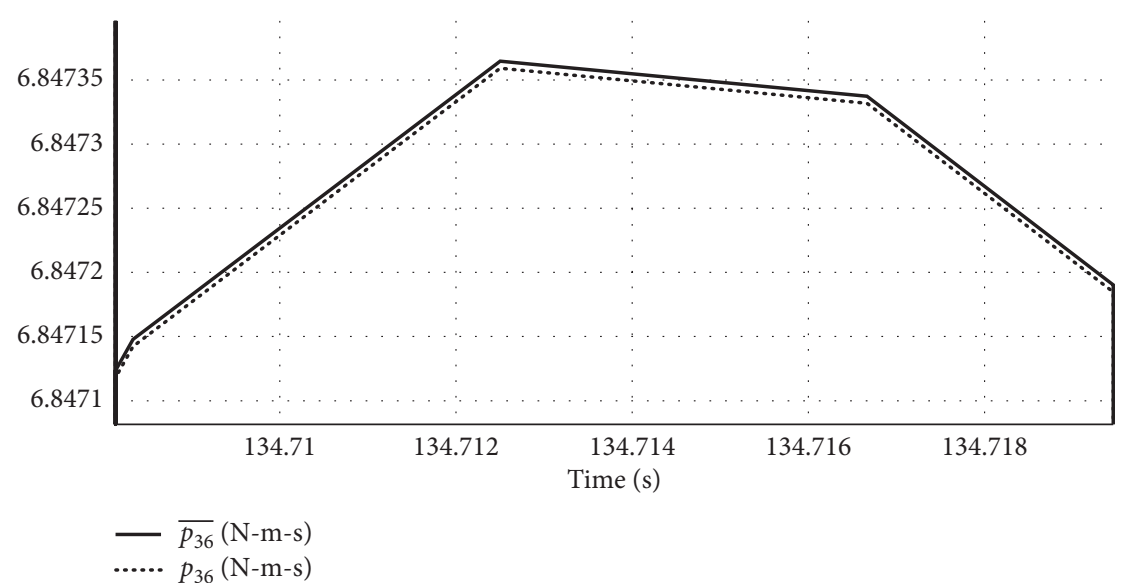

Figure 21: Close up of Figure 20.

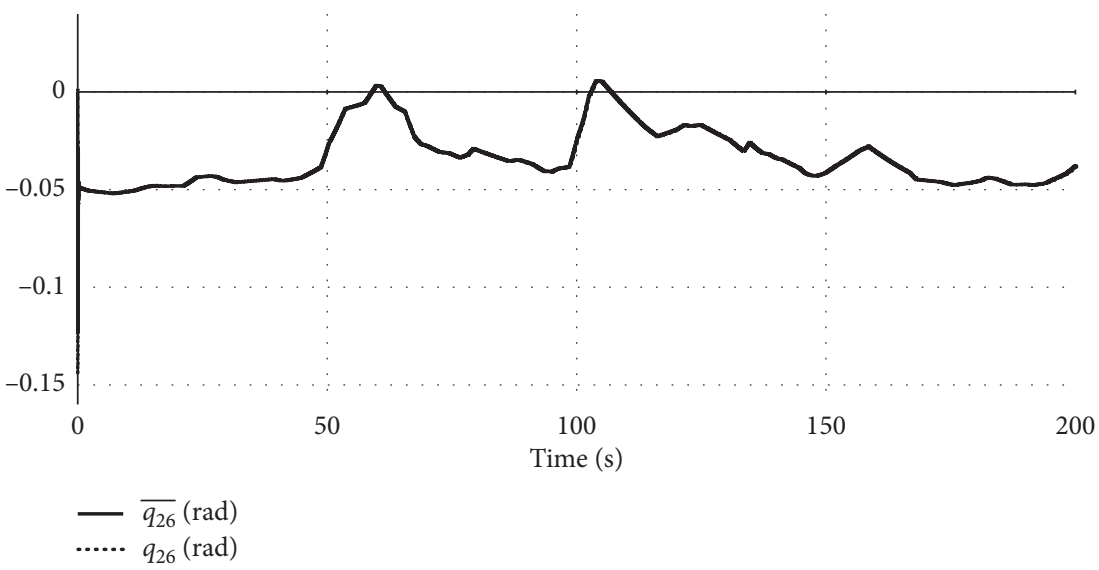

Figure 22: Full-time simulation of the slow variable $q_{26}$ and the quasi-steady state model $\overline{p_{26}}$.

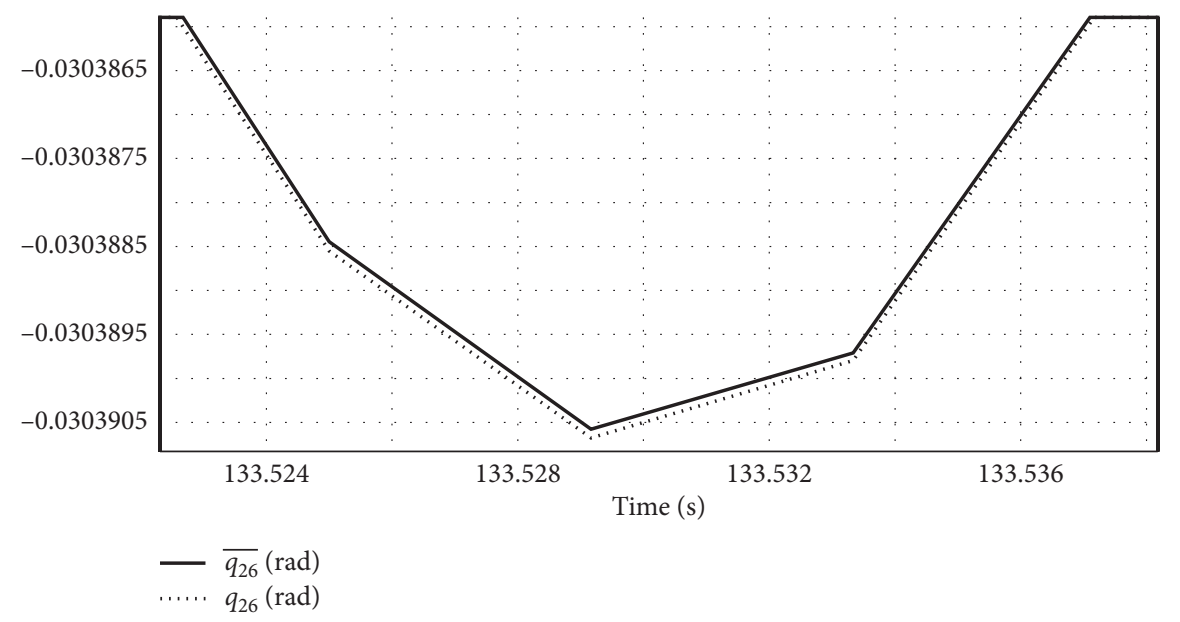

FIgURE 23: Close up of Figure 22. 


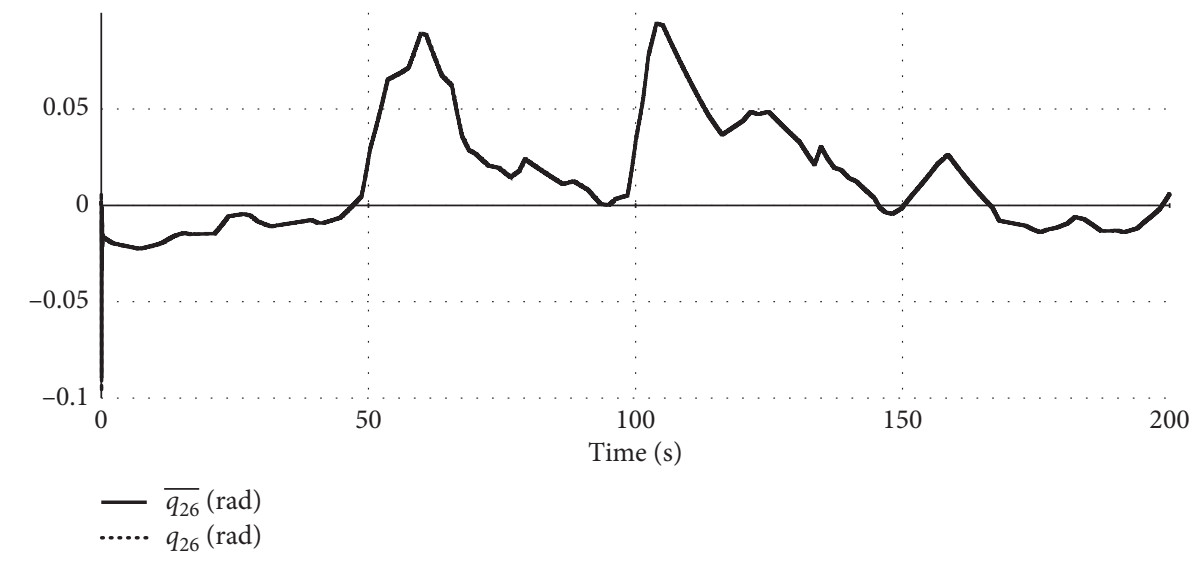

FIGURe 24: Full-time simulation of the slow variable $q_{33}$ and the quasi-steady state model $\overline{q_{33}}$.

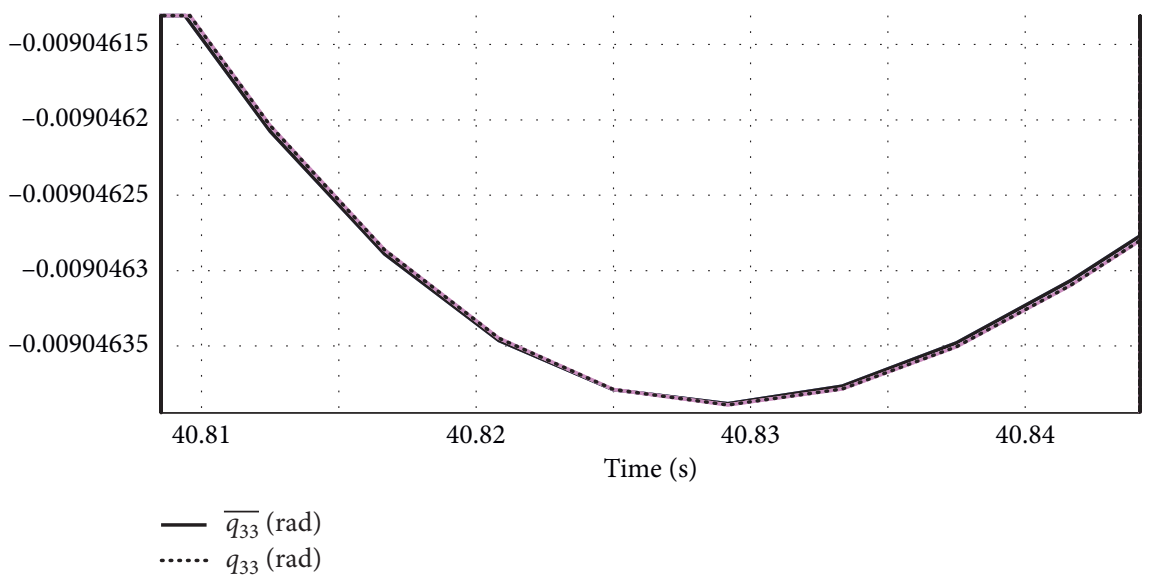

FIgURE 25: Close up of Figure 24.

\section{Conclusion}

(i) A class of singularly perturbed nonlinear systems modelled by bond graphs has been presented

(ii) The product of state variables and nonlinear dissipation elements represent the class of nonlinear systems of this paper

(iii) The state space representation of the complete nonlinear system of the bond graph with integral causality assignment for both dynamics is developed

(iv) When a derivative causality to the storage elements for the fast dynamics is assigned and the storage elements for the slow dynamics an integral causality is maintained, then the quasi-steady state model can be determined (v) The nonlinear physical system formed by a wind turbine connected to an induction generator has been modelled by bond graphs

(vi) The quasi-steady state model of this electromechanical system in the physical domain has been obtained

(vii) The performance for both dynamics based on simulation results has been shown

\section{Appendix}

\section{A. Proof of Lemma 1}

Proof. From the third and fourth lines of (17) with (15) and (16),

$$
\begin{aligned}
& D_{\text {in }}^{l}(t)=\left[I-S_{22}^{11}(x) L\right]^{-1}\left[S_{21}^{11}(x) z_{1}+S_{21}^{12}(x) z_{2}+S_{22}^{12}(x) D_{\text {out }}^{n l}(t)+S_{23}^{11}(x) u\right], \\
& D_{\text {in }}^{n l}(t)=\left[I-S_{22}^{22}(x) \phi_{L}\left(x_{1}\right)\right]^{-1}\left[S_{21}^{21}(x) z_{1}+S_{21}^{22}(x) z_{2}+S_{22}^{21}(x) D_{\text {out }}^{n l}(t)+S_{23}^{21}(x) u\right] .
\end{aligned}
$$


By substituting (A.2) into (A.1) with (35) and (35),

$$
\begin{aligned}
{\left[I-L^{-1} M_{L}(x) S_{22}^{12}(x) M_{\phi}(x) S_{22}^{21}(x) L\right] D_{\mathrm{in}}^{l}(t)=} & L^{-1} M_{L}(x)\left[S_{21}^{11}(x) z_{1}+S_{21}^{12}(x) z_{2}+S_{23}^{11}(x) u\right] \\
& +L^{-1} M_{L}(x) S_{22}^{12}(x) M_{\phi}(x)\left[S_{21}^{21}(x) z_{1}+S_{21}^{22}(x) z_{2}+S_{23}^{21}(x) u\right]
\end{aligned}
$$

where the term $\left[I-L^{-1} M_{L}(x) S_{22}^{12}(x) M_{\phi}(x) S_{22}^{21}(x) L\right]$ can be written by $L^{-1} M_{L}(x)\left[M_{L}^{-1}(x) L-S_{22}^{12}(x) M_{\phi}(x) S_{22}^{21}(x) L\right]$.
Equation (A.3) with (32) is given by

$$
\begin{aligned}
D_{\text {in }}^{l}(t)= & T_{M}(x)\left\{\left[S_{21}^{11}(x)+S_{22}^{12}(x) M_{\phi}(x) S_{21}^{21}(x)\right] z_{1}+\left[S_{21}^{12}(x)+S_{22}^{12}(x) M_{\phi}(x) S_{21}^{22}(x)\right] z_{2}\right. \\
& \left.+\left[S_{23}^{11}(x)+S_{22}^{12}(x) M_{\phi}(x) S_{23}^{21}(x)\right] u\right\} .
\end{aligned}
$$

From (A.1) and (A.2) with (35),

$$
\begin{aligned}
{\left[I-\phi_{L}^{-1}\left(x_{1}\right) M_{\phi}(x) S_{22}^{21}(x) M_{L}(x) S_{22}^{12}(x) \phi_{L}\left(x_{1}\right)\right] D_{\mathrm{in}}^{n l}(t)=} & \phi_{L}^{-1}\left(x_{1}\right) M_{\phi}(x)\left[S_{21}^{21}(x) z_{1}+S_{21}^{22}(x) z_{2}+S_{23}^{21}(x) u\right] \\
& +\phi_{L}^{-1}\left(x_{1}\right) M_{\phi}(x) S_{22}^{21}(x) M_{L}(x)\left[S_{21}^{11}(x) z_{1}+S_{21}^{12}(x) z_{2}+S_{23}^{11}(x) u\right]
\end{aligned}
$$

where the term $\left[I-\phi_{L}^{-1}\left(x_{1}\right) M_{\phi}(x) S_{22}^{21}(x) M_{L}(x) S_{22}^{12}(x)\right.$ $\left.\phi_{L}\left(x_{1}\right)\right]$ can be replaced by $\phi_{L}^{-1}\left(x_{1}\right) M_{\phi}(x)\left[M_{\phi}^{-1}(x) \phi_{L}\left(x_{1}\right)-\right.$ $\left.S_{22}^{21}(x) M_{L}(x) S_{22}^{12}(x) \phi_{L}\left(x_{1}\right)\right]$.

$$
\begin{aligned}
D_{\text {in }}^{n l}(t)= & T_{\phi S}(x)\left\{\left[S_{21}^{21}(x)+S_{22}^{21}(x) M_{L}(x) S_{21}^{11}(x)\right] z_{1}+\left[S_{21}^{22}(x)+S_{22}^{21}(x) M_{L}(x) S_{21}^{12}(x)\right] z_{2}\right. \\
& +\left[S_{23}^{21}(x)+S_{22}^{21}(x) M_{L}(x) S_{23}^{11}(x)\right] u .
\end{aligned}
$$

From the first and second lines of (17) with (A.4) and (A.6),

$$
\begin{aligned}
\dot{x}_{1}(t)= & \left\{S_{11}^{11}(x)+S_{12}^{11}(x) L T_{M}(x)\left[S_{21}^{11}(x)+S_{22}^{12}(x) M_{\phi}(x) S_{21}^{21}(x)\right]+S_{12}^{12}(x) \phi_{L}\left(x_{1}\right) T_{\phi}(x)\left[S_{21}^{21}(x)+S_{22}^{21}(x) M_{L}(x) S_{21}^{11}(x)\right] z_{1}\right\} \\
& +\left\{S_{11}^{12}(x)+S_{12}^{11}(x) L T_{M}(x)\left[S_{21}^{12}(x)+S_{22}^{12}(x) M_{\phi}(x) S_{21}^{22}(x)\right]+S_{12}^{12}(x) \phi_{L}\left(x_{1}\right) T_{\phi}(x)\left[S_{21}^{22}(x)+S_{22}^{21}(x) M_{L}(x) S_{21}^{12}(x)\right] z_{2}\right\} \\
& +\left\{S_{13}^{11}(x)+S_{12}^{11}(x) L T_{M}(x)\left[S_{23}^{11}(x)+S_{22}^{12}(x) M_{\phi}(x) S_{23}^{21}(x)\right]+S_{12}^{12}(x) \phi_{L}\left(x_{1}\right) T_{\phi}(x)\left[S_{23}^{21}(x)+S_{22}^{21}(x) M_{L}(x) S_{23}^{11}(x)\right] u\right\},
\end{aligned}
$$

$$
\begin{aligned}
\dot{x}_{2}(t)= & \left\{S_{11}^{21}(x)+S_{12}^{21}(x) L T_{M}(x)\left[S_{21}^{11}(x)+S_{22}^{12}(x) M_{\phi}(x) S_{21}^{21}(x)\right]+S_{12}^{22}(x) \phi_{L}\left(x_{1}\right) T_{\phi}(x)\left[S_{21}^{21}(x)+S_{22}^{21}(x) M_{L}(x) S_{21}^{11}(x)\right] z_{1}\right\} \\
& +\left\{S_{11}^{22}(x)+S_{12}^{21}(x) L T_{M}(x)\left[S_{21}^{12}(x)+S_{22}^{12}(x) M_{\phi}(x) S_{21}^{22}(x)\right]+S_{12}^{12}(x) \phi_{L}\left(x_{1}\right) T_{\phi}(x)\left[S_{21}^{22}(x)+S_{22}^{21}(x) M_{L}(x) S_{21}^{12}(x)\right] z_{2}\right\} \\
& +\left\{S_{13}^{21}(x)+S_{12}^{21}(x) L T_{M}(x)\left[S_{23}^{11}(x)+S_{22}^{12}(x) M_{\phi}(x) S_{23}^{21}(x)\right]+S_{12}^{22}(x) \phi_{L}\left(x_{1}\right) T_{\phi}(x)\left[S_{23}^{21}(x)+S_{22}^{21}(x) M_{L}(x) S_{23}^{11}(x)\right] u\right\} .
\end{aligned}
$$


From (20) to (31) with (A.7) and (A.8), equations (18) and (19) are proved.

\section{B. Proof of Lemma 2}

Proof. From the third and fourth lines of (38) with (39) and (40),

$$
\begin{aligned}
D_{\text {in }}^{h l}(t) & =\left[I-H_{22}^{11}(x) L^{h}\right]^{-1}\left[H_{21}^{11}(x) z_{1}(t)+H_{21}^{12}(x) \dot{x}_{2}(t)+H_{22}^{12}(x) D_{\text {out }}^{h n l}(t)+H_{23}^{11}(x) u(t)\right] \\
D_{\text {in }}^{h n l}(t) & =\left[I-H_{22}^{22}(x) \phi_{L}^{h}\left(x_{1}\right)\right]^{-1}\left[H_{21}^{21}(x) z_{1}(t)+H_{21}^{22}(x) \dot{x}_{2}(t)+H_{22}^{21}(x) D_{\text {out }}^{h n l}(t)+H_{23}^{21}(x) u(t)\right] .
\end{aligned}
$$

From (B.1) and (B.2) with (53),

$$
\begin{aligned}
{\left[I-\left(L^{h}\right)^{-1} Q_{L}(x) H_{22}^{12}(x) Q_{\phi}(x) H_{22}^{21}(x) L^{h}\right] D_{\text {in }}^{h l}(t)=} & \left(L^{h}\right)^{-1} Q_{L}(x)\left[H_{21}^{11}(x) z_{1}(t)+H_{21}^{12}(x) \dot{x}_{2}(t)+H_{23}^{11}(x) u(t)\right] \\
& +\left(L^{h}\right)^{-1} Q_{L}(x) H_{22}^{12}(x) Q_{\phi}(x)^{-1}\left[H_{21}^{21}(x) z_{1}(t)+H_{21}^{22}(x) \dot{x}_{2}(t)\right. \\
& \left.+H_{23}^{21}(x) u(t)\right]
\end{aligned}
$$

where the term $\left[I-\left(L^{h}\right)^{-1} Q_{L}(x) H_{22}^{12}(x) Q_{\phi}(x) H_{22}^{21}(x) L^{h}\right]$ is rewritten by $\left(L^{h}\right)^{-1} Q_{L}(x)\left[Q_{L}^{-1}(x) L^{h}-H_{22}^{12}(x) Q_{\phi}(x) H_{22}^{21}(x)\right.$ $\left.L^{h}\right]$; then,

$$
\begin{aligned}
D_{\text {in }}^{h l}(t)= & T_{Q}(x)\left\{\left[H_{21}^{11}(x)+H_{22}^{12}(x) Q_{\phi}(x) H_{21}^{21}(x)\right] z_{1}(t)+\left[H_{21}^{12}(x)+H_{22}^{12}(x) Q_{\phi}(x) H_{21}^{22}(x)\right] \dot{x}_{2}(t)\right. \\
& \left.+\left[H_{23}^{11}(x)+H_{21}^{12}(x) Q_{\phi}(x) H_{23}^{21}(x)\right] u(t)\right\} .
\end{aligned}
$$

By substituting (B.2) into (B.1) with

$$
\begin{aligned}
{\left[I-\left(\phi_{L}^{h}\left(x_{1}\right)\right)^{-1} Q_{\phi}(x) H_{22}^{21}(x) Q_{L}(x) H_{22}^{12}(x) \phi_{L}^{h}\left(x_{1}\right)\right] D_{\text {in }}^{h n l}(t)=} & \left(\phi_{L}^{h}\left(x_{1}\right)\right)^{-1} Q_{\phi}(x)\left[H_{21}^{21}(x) z_{1}(t)+H_{21}^{22}(x) \dot{x}_{2}(t)+H_{23}^{21}(x) u(t)\right] \\
& +\left(\phi_{L}^{h}\left(x_{1}\right)\right)^{-1} Q_{\phi}(x) H_{22}^{21}(x) Q_{L}(x)\left[H_{21}^{11}(x) z_{1}(t)+H_{21}^{12}(x) \dot{x}_{2}(t)\right. \\
& \left.+H_{23}^{11}(x) u(t)\right]
\end{aligned}
$$

the term $\left[I-\left(\phi_{L}^{h}\left(x_{1}\right)\right)^{-1} Q_{\phi}(x) H_{22}^{21}(x) Q_{L}(x) H_{22}^{12}(x) \phi_{L}^{h}\right.$

$\left.\left(x_{1}\right)\right]$ can be expressed by $\left(\phi_{L}^{h}\left(x_{1}\right)\right)^{-1} Q_{\phi}(x)\left[Q_{\phi}^{-1}(x)\right.$ $\left.\phi_{L}^{h}\left(x_{1}\right)-H_{22}^{21}(x) Q_{L}(x) H_{22}^{12}(x) \phi_{L}^{h}\left(x_{1}\right)\right]$; then, 


$$
\begin{aligned}
D_{\text {in }}^{h n l}(t)= & T_{\phi H}(x)\left\{\left[H_{21}^{21}(x)+H_{22}^{21}(x) Q_{L}(x) H_{21}^{11}(x)\right] z_{1}+\left[H_{21}^{22}(x)+H_{22}^{21}(x) Q_{L}(x) H_{21}^{12}(x)\right] \dot{x}_{2}(t)\right. \\
& \left.+\left[H_{23}^{21}(x)+H_{22}^{21}(x) Q_{L}(x) H_{23}^{11}(x)\right] u\right\} .
\end{aligned}
$$

From the first and second lines of (17) with (A.4) and (A.6),

$$
\begin{aligned}
& z_{2}(t)=\left\{H_{11}^{21}(x)+H_{12}^{21}(x) L^{h} T_{Q}(x)\left[H_{21}^{11}(x)+H_{22}^{12}(x) Q_{\phi}(x) H_{21}^{21}(x)\right]+H_{12}^{22}(x) \phi_{L}^{h}\left(x_{1}\right) T_{\phi H}(x)\right. \\
&\left.\cdot\left[H_{21}^{21}(x)+H_{22}^{21}(x) Q_{L}(x) H_{21}^{11}(x)\right] z_{1}(t)\right\} \\
&+\left\{H_{11}^{22}(x)+H_{12}^{21}(x) L^{h} T_{Q}(x)\left[H_{21}^{12}(x)+H_{22}^{12}(x) Q_{\phi}(x) H_{21}^{22}(x)\right]+H_{12}^{22}(x) \phi_{L}^{h}\left(x_{1}\right) T_{\phi H}(x)\right. \\
&\left.\cdot\left[H_{21}^{22}(x)+H_{22}^{21}(x) Q_{L}(x) H_{21}^{12}(x)\right] \dot{x}_{2}(t)\right\} \\
&+\left\{H_{13}^{21}(x)+H_{12}^{21}(x) L^{h} T_{Q}(x)\left[H_{23}^{11}(x)+H_{22}^{12}(x) Q_{\phi}(x) H_{23}^{21}(x)\right]+H_{12}^{22}(x) \phi_{L}^{h}\left(x_{1}\right) T_{\phi H}(x)\right. \\
&\left.\cdot\left[H_{23}^{21}(x)+H_{22}^{21}(x) Q_{L}(x) H_{23}^{11}(x)\right] u(t)\right\} \\
& \dot{x}_{1}(t)=\left\{H_{11}^{11}(x)+H_{12}^{11}(x) L^{h} T_{Q}(x)\left[H_{21}^{11}(x)+H_{22}^{12}(x) Q_{\phi}(x) H_{21}^{21}(x)\right]+H_{12}^{12}(x) \phi_{L}^{h}\left(x_{1}\right) T_{\phi H}(x)\right. \\
&\left.\cdot\left[H_{21}^{21}(x)+H_{22}^{21}(x) Q_{L}(x) H_{21}^{11}(x)\right] z_{1}(t)\right\} \\
&+\left\{H_{11}^{12}(x)+H_{12}^{11}(x) L^{h} T_{Q}(x)\left[H_{21}^{12}(x)+H_{22}^{12}(x) Q_{\phi}(x) H_{21}^{22}(x)\right]+H_{12}^{12}(x) \phi_{L}\left(x_{1}\right) T_{\phi H}(x)\right. \\
&\left.\cdot\left[H_{21}^{22}(x)+H_{22}^{21}(x) Q_{L}(x) H_{21}^{12}(x)\right] \dot{x}_{2}(t)\right\} \\
&+\left\{H_{13}^{11}(x)+H_{12}^{11}(x) L^{h} T_{Q}(x)\left[H_{23}^{11}(x)+H_{22}^{12}(x) Q_{\phi}(x) H_{23}^{21}(x)\right]+H_{12}^{12}(x) \phi_{L}^{h}\left(x_{1}\right) T_{\phi H}(x)\right. \\
&\left.\cdot\left[H_{23}^{21}(x)+H_{22}^{21}(x) Q_{L}(x) H_{23}^{11}(x)\right] u(t)\right\} .
\end{aligned}
$$

By substituting from (47) to (54) into (B.7) and (B.8) with (39) and (40), the components (43) and (44)-(46) are proved.

\section{Developed Junction Structure of equation (78)}

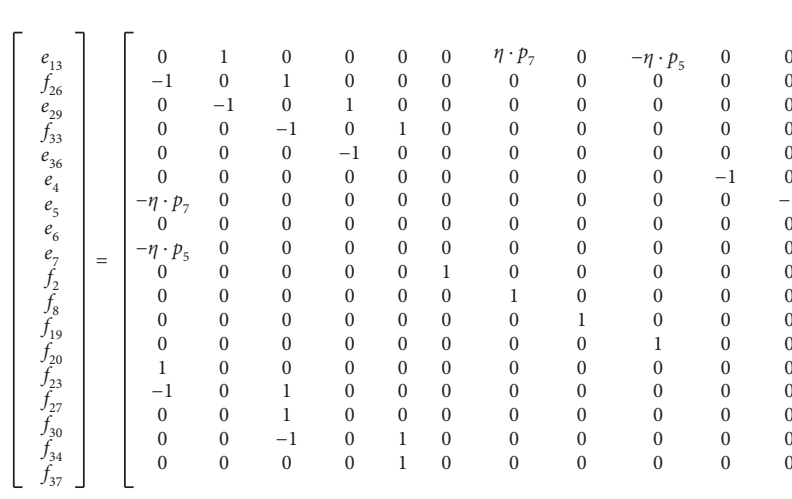

0

$\begin{array}{cc} & \\ 0 & -1 \\ 0 & 0 \\ 0 & 0 \\ 0 & 0 \\ 0 & 0 \\ 0 & 0 \\ 0 & 0 \\ 0 & 0 \\ -1 & 0 \\ 0 & 0 \\ 0 & 0 \\ 0 & 0 \\ 0 & 0 \\ 0 & 0 \\ 0 & 0 \\ 0 & 0 \\ 0 & 0 \\ 0 & 0 \\ & \end{array}$

$\begin{array}{ccc}0 & 0 & 0 \\ 0 & 0 & 0 \\ -1 & 1 & 0 \\ 0 & 0 & 0 \\ 0 & -1 & -1 \\ 0 & 0 & 0 \\ 0 & 0 & 0 \\ 0 & 0 & 0 \\ 0 & 0 & 0 \\ 0 & 0 & 0 \\ 0 & 0 & 0 \\ 0 & 0 & 0 \\ 0 & 0 & 0 \\ 0 & 0 & 0 \\ 0 & 0 & 0 \\ 0 & 0 & 0 \\ 0 & 0 & 0 \\ 0 & 0 & 0\end{array}$

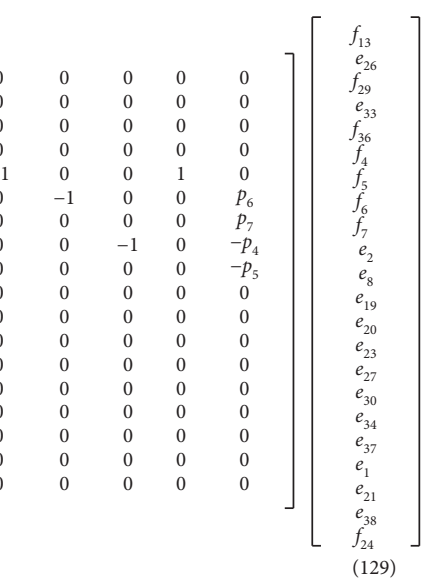

(C.1) 


\section{Developed Junction Structure of Figure 8}

$\left[\begin{array}{c}e_{13} \\ f_{26} \\ e_{29} \\ f_{33} \\ e_{36} \\ f_{4} \\ f_{5} \\ f_{6} \\ f_{7} \\ e_{2} \\ e_{8} \\ e_{19} \\ e_{20} \\ f_{23} \\ f_{27} \\ f_{30} \\ f_{34} \\ f_{37}\end{array}\right]=\left[\begin{array}{cccccc}0 & 1 & 0 & 0 & 0 & 0 \\ -1 & 0 & 1 & 0 & 0 & 0 \\ 0 & -1 & 0 & 1 & 0 & 0 \\ 0 & 0 & -1 & 0 & 1 & 0 \\ 0 & 0 & 0 & -1 & 0 & 0 \\ 0 & 0 & 0 & 0 & 0 & 0 \\ 0 & 0 & 0 & 0 & 0 & 0 \\ 0 & 0 & 0 & 0 & 0 & 0 \\ 0 & 0 & 0 & 0 & 0 & 0 \\ 0 & 0 & 0 & 0 & 0 & -1 \\ 0 & 0 & 0 & 0 & 0 & 0 \\ 0 & 0 & 0 & 0 & 0 & 0 \\ 0 & 0 & 0 & 0 & 0 & 0 \\ 1 & 0 & 0 & 0 & 0 & 0 \\ -1 & 0 & 1 & 0 & 0 & 0 \\ 0 & 0 & 1 & 0 & 0 & 0 \\ 0 & 0 & -1 & 0 & 1 & 0 \\ 0 & 0 & 0 & 0 & 1 & 0 \\ & & & & & \end{array}\right.$

$\begin{array}{cc}0 & 0 \\ 0 & 0 \\ 0 & 0 \\ 0 & 0 \\ 0 & 0 \\ 0 & 0 \\ 0 & 0 \\ 0 & 0 \\ 0 & 0 \\ 0 & 0 \\ -1 & 0 \\ 0 & -1 \\ 0 & 0 \\ 0 & 0 \\ 0 & 0 \\ 0 & 0 \\ 0 & 0 \\ 0 & 0\end{array}$

$\begin{array}{cc}0 & 0 \\ 0 & 0 \\ 0 & 0 \\ 0 & 0 \\ 0 & 0 \\ 0 & -1 \\ 0 & 0 \\ 0 & 0 \\ 0 & 0 \\ 0 & \\ 0 & 0 \\ 0 & 0 \\ -1 & 0 \\ 0 & 0 \\ 0 & 0 \\ 0 & 0 \\ 0 & 0 \\ 0 & 0\end{array}$

$\begin{array}{ccc}0 & \eta \cdot p_{7} & 0 \\ 0 & 0 & 0 \\ 0 & 0 & 0 \\ 0 & 0 & \\ 0 & 0 \\ -1 & 0 & 0 \\ 0 & -1 & 0 \\ 0 & 0 & -1 \\ 0 & 0 & \\ 0 & 0 \\ 0 & 0 \\ 0 & 0 \\ 0 & 0 \\ 0 & 0 \\ 0 & 0 \\ 0 & 0 \\ 0 & 0 \\ 0 & 0\end{array}$

0
0
0
0
0
0
0
-1
0
0
0
0
0
0
0
0
0
0

$-\eta \cdot p_{5}$
0
0
0
0
0
0
0
-1
0
0
0
0
0
0
0
0
0

$\begin{array}{cccc}-1 & 1 & 0 & 0 \\ 0 & 0 & 0 & 0 \\ 0 & -1 & -1 & -1 \\ 0 & 0 & 0 & 0 \\ 0 & 0 & 0 & -1 \\ 0 & 0 & 0 & 0 \\ 0 & 0 & 0 & 0 \\ 0 & 0 & 0 & 0 \\ 0 & 0 & 0 & 0 \\ 0 & 0 & 0 & 0 \\ 0 & 0 & 0 & 0 \\ 0 & 0 & 0 & 0 \\ 0 & 0 & 0 & 0 \\ 0 & 0 & 0 & 0 \\ 0 & 0 & 0 & 0 \\ 0 & 0 & 0 & 0 \\ 0 & 0 & 0 & 0 \\ 0 & 0 & 0 & 0\end{array}$

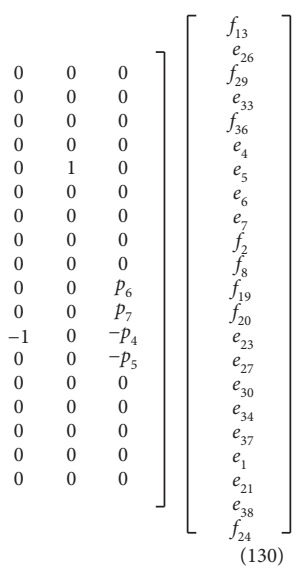

(D.1)

\section{Data Availability}

The data used to support the findings of this study have been sent by e-mail and were obtained using a turbine owned by the faculty of electrical engineering of the Michoacan University of San Nicolas de Hidalgo.

\section{Conflicts of Interest}

The authors declare that they have no conflicts of interest.

\section{References}

[1] D. S. Naidu and A. J. Calise, "Singular perturbations and time scales in guidance and control of aerospace systems-a survey," Journal of Guidance, Control, and Dynamics, vol. 24, no. 6, pp. 1057-1078, 2001.

[2] K. H. K. Kokotovic and J. O'Reilly, Singular Perturbation Methods in Control: Analysis and Design, Society for Industrial and Applied Mathematics, Philadelphia, PA, USA, 1999.

[3] L. S. Louca, J. L. Stein, and G. M. Hulbert, "Energy-based model reduction methodology for automated modeling," Journal of Dynamic Systems, Measurement, and Control, vol. 132, no. 6, pp. 1-16, 2010.

[4] B. Boukhezzar and H. Siguerdidjane, "Nonlinear control of variable speed wind turbines for power regulation," in Proceedings of the IEEE Conference on Control Applications, Toronto, ON, Canada, August 2005.

[5] N. Ullah, "Small scale integration of variable speed wind turbines into the local grid and its voltage stability aspects," in Proceedings of the International Conference on Future Power Systems, p. 8, Amsterdam, Netherlands, November 2005.

[6] J. G. Slootweg, H. Polinder, and W. L. Kling, "Dynamic modelling of a wind turbine with doubly fed induction generator," in Proceedings of the Power Engineering Society Summer Meeting Conference Proceedings Cat NoO1CH37262, vol. 1, pp. 644-649, Chicago, IL, USA, April 2001.

[7] R. Marino, P. Tomei, and C. M. Verrelli, Induction Motor Control Design, Springer, Berlin, Germany, 2010.
[8] A. M. Trzynadlowski, Control of Induction Motors, Academic Press Series in Engineering, Elsevier Science, Cambridge, Massachusettse, USA, 2001.

[9] R. E. Araujo, Induction Motors-Modelling and Control, InTech, London, UK, 2012.

[10] P. C. Krause and C. H. Thomas, "Simulation of symmetrical induction machinery," in Proceedings of the IEEE Transactions on Power Apparatus and Systems, vol. 84, no. 11, pp. 10381053, November 1965.

[11] D. C. Karnopp, D. L. Margolis, and R. C. Rosenberg, Modeling, Simulation, and Control of Mechatronic Systems, John Wiley \& Sons, Hoboken, NJ, USA, 2012.

[12] P. Wellstead, Introduction to Physical System Modelling.Academic Press, Cambridge, MA, USA, 2000.

[13] C. Sueur and G. Dauphin-Tanguy, "Bond-graph approach for structural analysis of MIMO linear systems," Journal of the Franklin Institute, vol. 328, no. 1, pp. 55-70, 1991.

[14] R. C. Rosenberg, "State-space formulation for bond graph models of multiport systems," Journal of Dynamic Systems, Measurement, and Control, vol. 93, no. 1, pp. 35-40, 1971.

[15] J. R. Ort and H. R. Martens, "The properties of bond graph junction structure matrices," Journal of Dynamic Systems, Measurement, and Control, vol. 95, no. 4, pp. 362-367, 1973.

[16] S. Junco and J. Jose, "Real-and complex-power bond graph modeling of the induction motor," in Proceedings of the International Conference; 4th, Bond graph modeling and simulation, pp. 323-330, San Francisco, CA, USA, 1999.

[17] C. Sueur and G. Dauphin-Tanguy, "Bond graph approach to multi-time scale systems analysis," Journal of the Franklin Institute, vol. 328, no. 5-6, pp. 1005-1026, 1991.

[18] G. Duphin-Tanguy and P. Borne, "Order reduction of multitime scale systems using bond graphs, the reciprocal system and the singular perturbation method," Journal of the Franklin Institute, vol. 319, no. 1, pp. 157-171, 1985.

[19] A. Gilberto Gonzalez and G. Noe Barrera, "Quasy steady state model determination using bond graph for a singularly perturbed LTI system," in Proceedings of the 16th International Conference On Methods \& Models In Automation \& Robotics 2011, pp. 194-199, Miedzyzdroje, Poland, August 2011.

[20] A. Gilberto Gonzalez, "A bond graph model of a singularly perturbed LTI MIMO system with a slow state estimated 
feedback," in Proceedings of the Institution of Mechanical Engineers, Part I: Journal of Systems and Control Engineering, vol. 230, no. 8, pp. 799-819, September 2016.

[21] G. Gonzalez and A. Padilla, "Approximate bond graph models for linear singularly perturbed systems," Mathematical and Computer Modelling of Dynamical Systems, vol. 22, no. 5, pp. 412-443, 2016.

[22] G. Gonzalez-Avalos and J. A. Padilla, "Quasi-steady-state model of a class of nonlinear singularly perturbed system in a bond graph approach," Electrical Engineering, vol. 100, no. 1, pp. 293-302, 2018.

[23] J. Buisson, H. Cormerais, and P.-Y. Richard, "Analysis of the bond graph model of hybrid physical systems with ideal switches," in Proceedings of the Institution of Mechanical Engineers, Part I: Journal of Systems and Control Engineering, vol. 216, no. 1, pp. 47-63, February 2002.

[24] R. Margetts, R. F. Ngwompo, and M. Fortes da Cruz, "Construction and analysis of causally dynamic hybrid bond graphs," in Proceedings of the Institution of Mechanical Engineers, Part I: Journal of Systems and Control Engineering, vol. 227, no. 3, pp. 329-346, March 2013.

[25] I. B. N. Al-Mashhadani and S. Hadjiloucas, "Bond-graph input-state-output port-Hamiltonian formulation of memristive networks for emulation of josephson junction circuits," Journal of Physics: Conference Series, vol. 1322, no. 1, p. 012040, 2019.

[26] D. Halliday, R. Resnick, and J. Walker, Fundamentals of Physics, John Wiley \& Sons, Hoboken, NJ, USA, 2010.

[27] H. Li and Z. Chen, "Transient stability analysis of wind turbines with induction generators considering blades and shaft flexibility," in Proceedings of the IECON Proceedings (Industrial Electronics Conference), pp. 1604-1609, Taipei, Taiwan, china, November 2007.

[28] H. Li, B. Zhao, C. Yang, H. W. Chen, and Z. Chen, “Analysis and estimation of transient stability for a grid-connected wind turbine with induction generator," Renewable Energy, vol. 36, no. 5, pp. 1469-1476, 2011.

[29] P. Vijay, A. K. Samantaray, and A. Mukherjee, "Bond graph modelling of a solid oxide fuel cell," in Bond Graph Modelling of Engineering Systems, W. Borutzky, Ed., Springer, New York, NY, USA, 2011.

[30] P. Krause, O. Wasynczuk, S. Sudhoff et al., "Analysis of Electric Machinery and Drive Systems," IEEE Press Series on Power Engineering, IEEE Press, Piscataway, NJ, USA, 2002. 